\title{
Spectroscopic ellipsometry and polarimetry for materials and systems analysis at the nanometer scale: state-of-the-art, potential, and perspectives
}

\author{
Maria Losurdo • Michael Bergmair - Giovanni Bruno - Denis Cattelan • \\ Christoph Cobet $\cdot$ Antonello de Martino $\cdot$ Karsten Fleischer • \\ Zorana Dohcevic-Mitrovic • Norbert Esser • Melanie Galliet • \\ Rados Gajic · Dušan Hemzal · Kurt Hingerl · Josef Humlicek • \\ Razvigor Ossikovski · Zoran V. Popovic · Ottilia Saxl
}

Received: 23 January 2009/Accepted: 19 May 2009/Published online: 12 June 2009

(C) Springer Science+Business Media B.V. 2009

\begin{abstract}
This paper discusses the fundamentals, applications, potential, limitations, and future perspectives of polarized light reflection techniques for the characterization of materials and related systems and devices at the nanoscale. These techniques include spectroscopic ellipsometry, polarimetry, and reflectance anisotropy. We give an overview of the various ellipsometry strategies for the measurement and analysis of nanometric films, metal nanoparticles and nanowires, semiconductor nanocrystals, and submicron periodic structures. We show that ellipsometry is capable of more than the determination of thickness and optical properties, and it can be exploited to gain information about process control,
\end{abstract}

\footnotetext{
M. Losurdo $(\bowtie) \cdot$ G. Bruno

CNR-IMIP, National Council of Research-Institute

of Inorganic Methodologies and of Plasmas,

Bari, Italy

e-mail: maria.losurdo@ba.imip.cnr.it

M. Bergmair · K. Hingerl

Christian Doppler Laboratory for Surface Optics, Center

for Surface- and Nanoanalytics, Johannes Kepler

University Linz, Linz, Austria

D. Cattelan - M. Galliet

Thin Film Division, HORIBA Scientific, Chilly Mazarin, France

e-mail: denis.cattelan@horiba.com

M. Galliet

e-mail: melanie.gaillet@horiba.com
}

geometry factors, anisotropy, defects, and quantum confinement effects of nanostructures.

Keywords Spectroscopic ellipsometry · Polarimetry · Nanomaterials · Nanoparticles · Thin films - Optical characterization . Nanometrology $\cdot$ Review

\section{Introduction}

The synthesis of nanomaterials, the fabrication of structures at the nanometer scale, and the control of

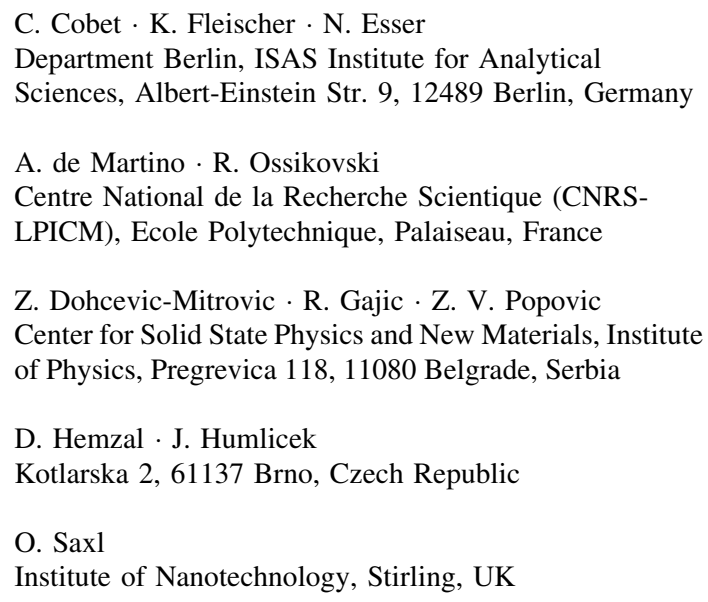


nanoscale systems have become increasingly active areas for materials science, affecting many technological fields ranging from nanomanufacturing and micro/nano-robotics to devices for biotechnology, information technology, energy saving, and medicine. Various experimental approaches, both topdown and bottom-up, from the assembly of nanosized clusters to chemical etching and ion beam lithography, are being exploited to produce nanostructured materials. Undoubtedly, the development of nanotechnology has been spurred on by the refinement of measurement and diagnostic tools able to measure materials and systems at the nanoscale. Indeed, fully comprehensive nanomaterials characterization needs to address, understand, and tailor the interplay between the nanostructure, its properties, and its functionality. Understanding the chemistry and physics behind the formation of nanostructures, which essentially originate from the confinement effect of the dimension at the nanoscale and of the size/shape of the nanostructure, is of basic and technological importance. The ability to characterize materials and elucidate structures at the nanoscale will be critical to the advancement of nanotechnology, as will the ability to monitor the processes leading to those nanostructures, at the nanoscale in real time. These capabilities are mandatory for the successful development of nanotechnology and require in situ monitoring, measurement, and control of the nanoscale processes. For example, semiconductor manufacturing, which is exponentially moving to the nanoscale, increasingly requires measurement techniques that can evaluate features at tens of nanometers, nondestructively and in-line. Nonimaging, optical critical dimension techniques are expected to be key technologies in current and future semiconductor manufacturing processes, as cited in the most recent version of the International Technology Roadmap for Semiconductors (http://www.itrs.net). Therefore, metrology and reliable characterization methodologies, together with innovative synthesis methodologies, are fundamental to nanotechnology at all levels.

Well-established microscopy techniques, including atomic force microscopy (AFM), scanning electron microscopy (SEM), and transmission electron microscopy (TEM), have allowed the visualization of nanostructures, although they suffer from being "local," intrusive, and unsuitable for real-time, inline monitoring of processes and surface/interface modifications. Among the various characterization tools, spectroscopic ellipsometry (SE), which is a nondestructive, nonintrusive, and noninvasive, contactless optical technique, has been developed and applied extensively over the last 50 years, not only for the optical characterization of bulk materials and thin films, but also for in situ real-time measurement of multilayered film structures, interfaces, and composites, during fabrication and processing (Archer 1964; Tompkins and Irene 2005). Spectroscopic ellipsometry became famous for its extreme sensitivity to very thin overlayers well below $1 \mathrm{~nm}$ thickness (Tompkins and Irene 2005). Ellipsometry is routinely used to measure thickness and optical constants of dielectric, semiconductor, and metal thin films, and recently Hilfiker et al. (2008) have provided an overview discussing critically how to reduce the correlation between film thickness and optical constants. Going to the nanometer scale, since the first communication on the formation of self-assembled monolayers of an organic molecule in 1988, ellipsometry has been used for the measurement of self-assembly monolayers (SAMs) with a thickness of few tens of nanometers (Wasserman et al. 1989). Going further to a scale of $<1 \mathrm{~nm}$, although fashionable when framed in the new context of nanotechnology, the use of ellipsometry for the real-time investigation of adsorption kinetics of gases down to single atom layers (Carroll and Melmed 1969), as well as of proteins on surfaces below the monolayer coverage (Hall 1966; Cuypers et al. 1983), is not a new technique.

Spectroscopic ellipsometry offers much more than measuring thickness, namely, it has evolved into an efficient characterization tool for bulk materials, thin films, and stacked layered systems. For such systems, information provided by this technique is very rich, since it enables the accurate measurement of surface roughness and interfaces, while the determination of the complex refractive index gives access to fundamental physical parameters and a variety of sample properties, including morphology, crystallinity, chemical composition, and electrical conductivity. The main benefit of ellipsometric measurements, compared with other optical measurements, is the direct evaluation of both the real and imaginary parts of the complex dielectric function $\varepsilon=\varepsilon_{1}+\mathrm{i} \varepsilon_{2}$ [which relates to the refractive index, $n$, and extinction coefficient, $k$, $\left.\varepsilon=(n+\mathrm{ik})^{2}\right]$, through the ellipsometric angles $\Psi$ and $\Delta$ (see the Section on "Basics" below), with no 
need for Kramers-Kronig transforms. The determination of the complex dielectric function enables the investigation of a material's electronic structure, which is directly related to the joint density of states for interband absorption. This electronic structure and consequently the determined dielectric function are very sensitive to the materials' nanostructure (since nanocrystallites may induce quantum-size effects), to the point where defects may create localized states within the band gap, to the various phases present in nanocomposites materials, i.e., nanocermets (Toudert et al. 2008), which may have different electronic structures band gaps, and to the anisotropy generated in nanowires and nanorods by geometric asymmetry, etc. Therefore, ellipsometry can also provide useful information about nanostructures, such as the effective dielectric constants and the geometric structure.

As an example, generalized ellipsometry has been used to measure not only the optical properties but also the inclination angle of nanorods (Beydaghyan et al. 2005). The size effects at the nanoscale are very pronounced in semiconductor heterostructures, where the electronic states can be tailored just by selecting a proper geometry and sizes in quantum wells (Santos et al. 1994), quantum wires, and quantum dots (Gallas and Rivory 2003).

A need to noninvasively probe thin films early in their processing, as well as the economic drive to extend the capabilities of existing in-line platforms, has led us to explore optical methods as the basis for acquiring a complete understanding of physical, optical, and electrical properties at the atomic scale. There is also the desire to exploit real-time SE techniques for research and in-line production, stemming from the ability to discern the surface, interface, and bulk characteristics of the individual thin films that constitute a complex multilayer stack. In fact, many processes such as film nucleation and growth, oxidation, nitridation are slow and thus can be readily followed using ellipsometry. In recent years, advances in hardware and software have improved the speed of SE to the point where real-time processes can now be measured. This ability, when combined with the high sensitivity of SE to submonolayer changes in thin films, makes it a powerful method for noninvasive investigations of thin film dynamics.

As an example, the use of SE in real-time diagnostics for metalorganic vapor phase epitaxy of
III-V materials has been reviewed by Aspnes (2005). The application of real-time SE to elucidate Si-based thin film deposition processes in a reactive environment, like plasma enhanced chemical vapor deposition (PECVD), and to identify phase transitions from amorphous to nanocrystalline and microcrystalline growth regimes and control Si-based film nanostructures has been well established by Collins et al. (2003). Furthermore, additional examples of SE realtime control of nucleation and heteroepitaxial growth of III-Nitrides (e.g., GaN, InN, etc.) by molecular beam epitaxy are provided by Losurdo et al. (2005) and Cobet et al. (2003), giving evidence of the versatility of real-time $\mathrm{SE}$ to monitor (detect) at the nanoscale, independent of the deposition methodology and material systems.

Although the cited examples indicate that since the 1970s ellipsometry has been mainly exploited in microelectronics, in optics and optoelectronics, and in biomedicine, for measuring the thicknesses of a few tens of nanometers, new nanotechnologies, such as the recent, appealing plasmonics (Maier 2007), can also benefit from the use of SE. Nanometer-sized metal particles, rods, and wires have recently attracted much scientific interest due to their promising applications in photonics, photovoltaics, catalysis, ultrahigh data storage, and biomolecular sensors. There are various reports in the literature on the optical absorption spectra of nanoparticles of noble metals, mainly gold (Dalacu and Martinu 2000; Kooij et al. 2002; Scaffardi and Tocho 2006) and silver (Wormeester et al. 2006; Oates 2006) characterized by localized surface plasmon resonance (SPR). For spherical nanoparticles isolated or embedded in an isotropic medium, the Mie theory (Kreibig and Vollmer 1995) has been applied to describe the localized plasmon resonance peak. However, it is still challenging to quantify the optical properties of highdensity nanoparticles whose electric fields overlap, influencing the plasmon resonance frequency, which is the situation of nanoparticles supported on a surface (Haes et al. 2004; Wessels et al. 2004). This opens new questions also on the measurement and analysis of the plasmonic optical response of substrate-supported metal nanoparticles, since nanoparticles, depending on the photonic device, might be deposited on absorbing substrates, precluding common transmittance measurements. In this frame, plasmonic ellipsometry has also shown a great 
potential for measuring and tuning the plasmonic properties of metal nanoparticles supported on various substrates in real time (Wu et al. 2007, 2009; Giangregorio et al. 2006).

Therefore, ellipsometry is a versatile and very sensitive optical technique, with unequalled capabilities for addressing the correlation between processchemistry-structure-electronic properties-optical properties for a large variety of materials and nanostructures. This raises the following questions:

- what can be measured by ellipsometry at the nanoscale?

- how reliable and accurate is ellipsometry at the nanoscale?

- what is specifically required to go from the microto the nanoscale?

We aim to answer the above questions by discussing recent trends in the application of spectroscopic ellipsometry and polarimetry techniques for the characterization of materials and related systems and devices at the nanoscale, and give an overview of the various ellipsometry strategies. Three principal trends that provide the organizational framework for the paper are identified as follows:

1. Undertaking the characterization research required for determining the correlation between the nanostructure and the optical-chemical properties of the nanomaterial; the optical analysis of nanomaterials, from semiconductors and oxide nanocrystals to metal nanoparticles and nanowires, by spectroscopic ellipsometry and reflection anisotropy spectroscopy.

2. Developing methods for generating novel nanomaterials and innovative processes for fabricating nanostructured materials and devices with tailored performances. In this frame, in situ ellipsometry with subnanometer depth resolution is exploited for investigating the dynamics of surfaces, thin films, nanomaterials, and nanostructures during synthesis and processing at the nanoscale. Understanding and controlling the process parameters for nanomaterials growth during synthesis are critical in the successful fabrication of materials with tailored properties.

3. Enhancing measurement capabilities and developing models for rationalizing the properties and improving the knowledge of periodic nanosystems. As an example, the metrology of submicron gratings is presented.

The paper is organized as follows: Section "Fundamentals" provides the ellipsometry basics. Section "Ellipsometry Capabilities for Measurements at the Nanoscale" discusses capabilities for measurements at the nanoscale for the characterization of nanometric films and monitoring of relative growth and/or processing. Section "What can ellipsometry do for nanoparticles, nanocrystals, and nanowires?" presents and discusses ellipsometry and reflectance anisotropy spectroscopy (RAS) applications to metal nanoparticles, metal nanowires, and nanocrystals characterization, with the focus on quantum confinement and nanostructure-induced anisotropy effects. In Section "Metrology of Submicrometer Gratings", implementation of spectroscopic ellipsometry by Mueller polarimetry is presented through the example of the nondestructive characterization of periodic structures (1D or 2D gratings) with submicrometer characteristic dimensions. This is a task of major importance for process control in microelectronics, as well as other applications. In this respect, innovative techniques such as spectrally and angularly resolved Mueller polarimetry in conical diffraction may prove useful alternatives to classical ellipsometry in assessing the relevance of the theoretical model and reducing parameter correlations. Finally, Section "Limitations, implementations, and future trends" discusses present limitations, new trends and directions, and future prospects and challenges for basic science and technology as well as presents perspectives from instrumentation and market/technology.

\section{Fundamentals}

\section{Ellipsometry basics}

Spectroscopic ellipsometry is based on measuring the change in the polarization state of a linearly polarized light reflected from a sample surface, as schematically shown in the top panel of Fig. 1. Specifically, for a film or nanostructure growing along the negative $z$ direction (see the situation depicted in the bottom panel of Fig. 1), the structure and associated process is monitored using the polarized light with the plane of incidence $(x, z)$ incident obliquely at the sample surface. The angle of incidence, $\phi_{0}$, which is 

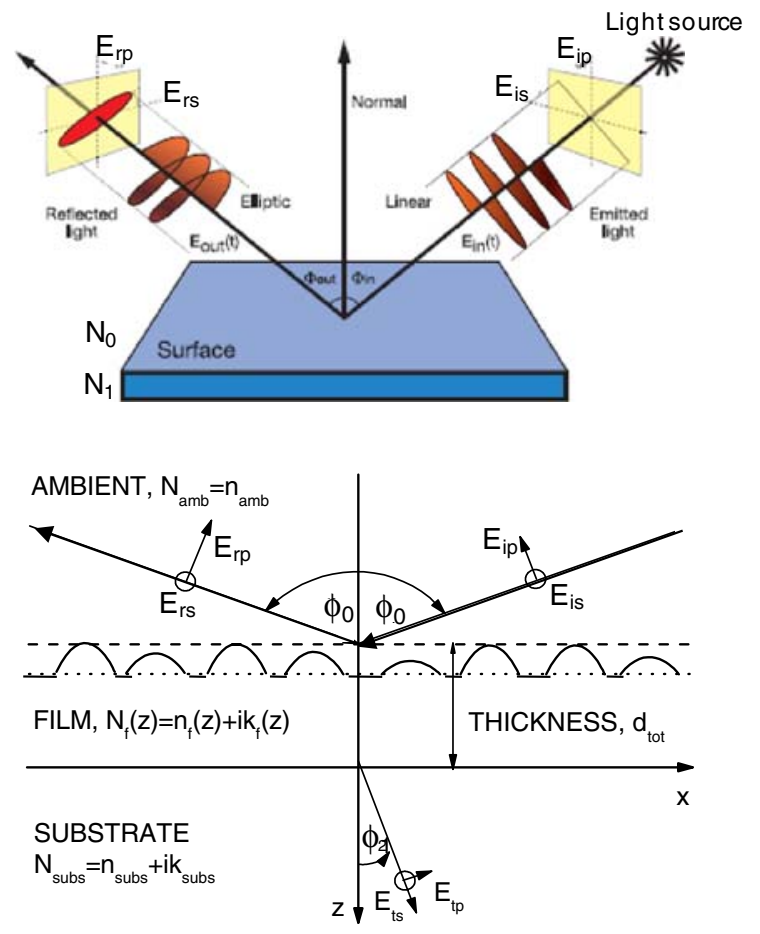

Fig. 1 Top panel: Scheme of reflection of linearly polarized light at a substrate/ambient interface; N0 and N1 are the complex refraction index of the ambient and substrate (as better specified in the bottom panel). Bottom panel: Reflection and transmission of a polarized plane wave at the planar ambient-film-substrate system. Nonideal surface morphology is shown schematically

typically $70^{\circ}$ for semiconductors, should be chosen carefully, depending on the Brewster angle of the materials under investigation (Tompkins and Irene 2005; Azzam and Bashara 1977). The interaction of polarized light with a sample can be represented by the Jones matrix formalism (Azzam and Bashara 1977)

$$
\left[\begin{array}{c}
E_{\mathrm{p}}^{\mathrm{r}} \\
E_{\mathrm{s}}^{\mathrm{r}}
\end{array}\right]=\left[\begin{array}{cc}
\tilde{R}_{\mathrm{pp}} & \tilde{R}_{\mathrm{sp}} \\
\tilde{R}_{\mathrm{ps}} & \tilde{R}_{\mathrm{ss}}
\end{array}\right] \cdot\left[\begin{array}{c}
E_{\mathrm{p}}^{\mathrm{i}} \\
E_{\mathrm{s}}^{\mathrm{i}}
\end{array}\right]
$$

where $E_{\mathrm{p}}^{\mathrm{i}}, E_{\mathrm{s}}^{\mathrm{i}}, E_{\mathrm{p}}^{\mathrm{r}}$, and $E_{\mathrm{s}}^{\mathrm{r}}$ represent the parallel (p) and perpendicular (s) components of the incident (i) and reflected (r) electric field of the light beam; $R_{\mathrm{pp}}$ and $R_{\mathrm{ss}}$ are the complex Fresnel reflection coefficients of the sample for p- and s-polarized light, respectively, defined for the simple ambient/material case in the top panel of Fig. 1 as

$$
\begin{aligned}
\tilde{R}_{\mathrm{pp}} & =r_{\mathrm{p}}=\frac{E_{\mathrm{p}}^{\mathrm{r}}}{E_{\mathrm{p}}^{\mathrm{i}}}=\frac{\tilde{N}_{1} \cos \phi_{0}-\tilde{N}_{0} \cos \phi_{1}}{\tilde{N}_{1} \cos \phi_{0}+\tilde{N}_{0} \cos \phi_{1}} \\
& =\left|r_{\mathrm{p}}\right| \mathrm{e}^{\mathrm{i} \delta_{\mathrm{p}}} \text { and } \\
\tilde{R}_{\mathrm{ss}} & =r_{\mathrm{s}}=\frac{E_{\mathrm{s}}^{\mathrm{r}}}{E_{\mathrm{s}}^{\mathrm{i}}}=\frac{\tilde{N}_{1} \cos \phi_{0}-\tilde{N}_{1} \cos \phi_{1}}{\tilde{N}_{0} \cos \phi_{0}+\tilde{N}_{1} \cos \phi_{1}}=\left|r_{\mathrm{s}}\right| \mathrm{e}^{\mathrm{i} \delta_{\mathrm{s}}}
\end{aligned}
$$

where $\delta_{\mathrm{p}}$ is the phase change for the $\mathrm{p}$ component upon reflection and similarly for the s component. The off-diagonal terms $R_{\mathrm{ps}}$ and $R_{\mathrm{sp}}$ describe the crosscoupling of the p- and s-polarized light and are zero for an isotropic sample. Thus, ellipsometric measurements are normally described by two parameters $\Psi$ and $\Delta$ in the following form:

$\rho=\frac{r_{\mathrm{p}}}{r_{\mathrm{s}}}=\tan \Psi \mathrm{e}^{\mathrm{i} \Delta}$

where $\tan \psi=\left|r_{\mathrm{p}}\right| /\left|r_{\mathrm{s}}\right|$ or $\left|\tilde{R}_{\mathrm{pp}}\right| /\left|\tilde{R}_{\mathrm{ss}}\right|$ is the amplitude ratio upon reflection and $\Delta=\delta_{\mathrm{p}}-\delta_{\mathrm{s}}$ is the phase shift difference. The ratio $\rho$ is related to the optical properties of the material under investigation, namely, the complex dielectric function, $\varepsilon=\varepsilon_{1}+i \varepsilon_{2}$, the refractive index, $n$, and the extinction coefficient, $k$, by the equation

$$
\begin{aligned}
\varepsilon & =\sin ^{2} \phi_{0}+\sin ^{2} \phi_{0} \tan ^{2} \phi_{0}\left[\frac{1-\rho}{1+\rho}\right]^{2}=N^{2} \\
& =(n+\mathrm{ik})^{2} .
\end{aligned}
$$

The high sensitivity of the technique is derived from the fact that the measurement of $\Psi$ and $\Delta$ is a relative measurement of the change in polarization (a ratio or difference of two values rather than the absolute value of either). It explains the robustness, the high accuracy, and the reproducibility of the technique. For instance, unlike absolute light intensity measurement, ellipsometry is relatively insensitive to scatter and fluctuations, and requires no standard sample or reference beam. Because spectroscopic ellipsometry also measures two values ( $\Psi$ and $\Delta)$ at each wavelength, this technique obtains more information than standard optical reflection techniques. The information learned is greatly enhanced by using wavelengths over a wide spectral range, from vacuum ultraviolet to mid-infrared. The far UV is the spectral range most sensitive to small changes, such as ultrathin layers or interfaces and films with low index contrast, gradient, and anisotropy. The NIR spectral range is necessary to determine the thickness 
of materials that are strongly absorbed in the visible spectrum.

Spectroscopic ellipsometer hardware

All spectroscopic ellipsometers begin with a white light source used to illuminate the sample (see scheme in Fig. 2). The light is passed through a first polarizer. When the polarized light reflects from the sample, both the phase and amplitude of the components describing the light can change. The exact quantification of the phase change, called $\Delta$, and the amplitude change, called $\Psi$, is determined by the sample's properties (thickness, complex refractive index).

This polarization change is analyzed by a second polarizer called an analyzer. An optional compensator (retarder, photoelastic modulator) can be placed in the path of the incident or reflected light beam. The addition of such an optical component is important to increase the accuracy of the ellipsometer by measuring accurate $(\Psi, \Delta)$ on its whole definition range. It is important to notice that one of these optical elements is modulated either mechanically (by rotation) or piezo-electrically. The polarization modulation provides the advantage of measuring an intensity ratio, instead of pure intensity. Therefore, ellipsometry is less affected by intensity instabilities. Finally the light falls onto the detector, which is commonly one of three types: photomultiplier, semiconductor photodiode, or CCD array. Spectroscopic ellipsometers measure the variation of intensity received on the

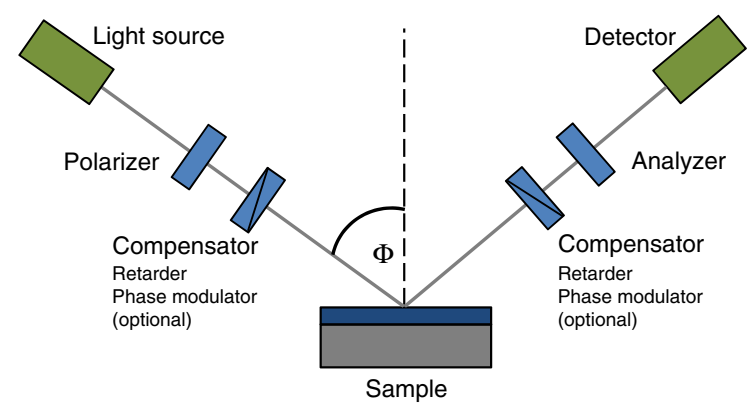

Fig. 2 Basic scheme of an ellipsometer components: a white light source used to illuminate the sample, a polarizer, a compensator (retarder, photoelastic modulator) that can be placed in the path of the incident or reflected light beam, a second polarizer called analyzer, and the detector detector synchronized with the polarization modulation.

The accuracy of an ellipsometer and its whole hardware design, including optical components and detectors, is often defined by the air measurement in straight-through configuration. Current $(\Psi, \Delta)$ technical specifications are around $\Psi \pm 0.08^{\circ}$ and $\Delta \pm 0.1^{\circ}$ taken on the spectral range visible-NIR. It is also important to notice that multi-angle measurements may enhance the accuracy of results, if the angles are judiciously chosen to maximize the $(\Psi, \Delta)$ sensitivity of the sample under study. It is useless, even dangerous, to add nonsensitive $(\Psi, \Delta)$ data; it is much better to use an accurate measurement at a unique angle of incidence.

\section{Ellipsometry capabilities for measurements at the nanoscale}

Scaling effect to nanodimension

The fundamental reason of the applicability of ellipsometry to the analysis of nanostructures and nanometric films stems from the fact that the optical properties of the materials, especially semiconductors, 'fingerprinted' by their dielectric function (Tompkins 1993; Yu and Cardona 1999; Lautenschlager et al. 1987), are modified by quantum confinement and surface states induced by their nanodimensionality. The dielectric function is characterized by critical points (CPs) in the VIS-VUV spectral range where electrons are excited from the top of a valence band to the conduction band. Absorption is strongest at CPs, where the bands are nearly parallel. Quantum confinement, in one, two, or three dimensions, changes the energy of the critical points and the joint density of states and, consequently, the dielectric function of nanomaterials, whose amplitude also depends on the characteristic size of the nanostructure. A well-known and largely investigated example is silicon, whose dependence of dielectric function on particle size (Jellison et al. 1993) down to $<3 \mathrm{~nm}$ nanocrystallite size has been reported in the literature (Losurdo et al. 2003). It is also interesting to note the emergence of strong anisotropy in silicon nanowires less than $2.2 \mathrm{~nm}$ in diameter and the appearance of new low-energy 
absorption peaks for light polarization along the wire axis (Zhao et al. 2004).

Furthermore, the nanostructuring of the surface/ film/material strongly modifies the polarizationdependent optical response. As an example, asymmetric and anisotropic shapes of nanostructures also lead to anisotropy in the optical response. Ellipsometric characterization of such nanostructures is possible but requires generalized ellipsometry (Jellison and Modine 1997) or Mueller matrix ellipsometry (Laskarakis et al. 2004; De Martino et al. 2008; Novikova et al. 2006). Mueller matrix ellipsometry offers the great advantage that depolarizing samples can also be analyzed, where depolarization may arise from irregularities in the nanostructure (shape, size, and ordering) and from multiple scattering. An example in the literature is given in the ellipsometric analysis of nanocones of GaSb (Nerbø et al. 2008). For anisotropic nanostructures, information on anisotropy, which is not limited to the optical response but also affects functional properties, can also be complemented by the RAS (Aspnes 1982; Shkrebtii et al. 1998). An interesting example is given in Section "What can ellipsometry do for nanoparticles, nanocrystals, and nanowires?" where, using the example of metallic nanowires, it is shown that appropriate modeling of the anisotropy in the measured optical spectra of metallic nanowires can be related to the conductance anisotropy, thus facilitating contactless measurements of this quantity. Additionally, for nanometric very thin films $<5 \mathrm{~nm}$, including thermally grown $\mathrm{SiO}_{2}$, it has been found that the real part of the refractive index $(n)$ changes with film thickness, $L_{\mathrm{ox}}$ (Grunthaner and Grunthaner 1986), and the assumption of refractive index of bulk or thin layers is no longer valid. As an example, for 1.4-8-nm-thick $\mathrm{SiO}_{2}$ films, a formula for $n$ for $\mathrm{SiO}_{2}$ (Wang and Irene 2000) was obtained as

$n=2.139-8.991 \times 10^{-2} L_{\mathrm{ox}}+1.872 \times 10^{-3} L_{\mathrm{ox}}^{2}$.

It was observed that as the oxide thickness increases, the value of $n=1.465$, which is the bulk $\mathrm{SiO}_{2}$ refractive index, is reached at around $30 \mathrm{~nm}$. A consequence of using the bulk $\mathrm{SiO}_{2}$ value of $n=1.465$ for a $5 \mathrm{~nm} \mathrm{SiO}$ film is a wrong thicknesses estimation, $20 \%$ larger than the real one. This underlines the importance of using ellipsometry in conjunction with an independent measurement of thickness for assessing reliable optical properties at the nanoscale.

As another significant scaling effect, nanoscale mechanisms have faster dynamics. Thus, the timescales of phenomena and reactivity can become very short, requiring fast nonintrusive techniques for detecting the dynamics and kinetics of chemical and structural modifications at the nanoscale. The following paragraphs and section discuss examples of how ellipsometry can deal with scaling effects related to the nanodimension.

How accurate is ellipsometry for nanometric thickness measurement?

Ultra-thin films have varied applications in optics, microelectronics, coating technologies, biomedicine, and wear protection. These technologies and related systems require the investigation of nucleation and growth processes, solid state reactions, the chemical, thermal, and mechanical stability of the thin film and interfaces, and the dynamics of their modifications as a function of film thickness. Therefore, thickness is an important parameter for all thin film and coating technologies, but it is especially demanding in microelectronics, which requires continuous reduction of the component sizes which have entered the nanoscale in 2000. In the following paragraph, the accuracy of ellipsometry for the determination of below-nanometer-size layer thicknesses is discussed.

The sensitivity of ellipsometry can be quantified by the calculation of the effect of the presence of a layer on a substrate on the parameters $(\Psi, \Delta)$. Table 1 shows calculated results for a c-Si substrate (with $n=3.8819$ and $k=0.019$ at $633 \mathrm{~nm}$ ) coated with a transparent film with $n=1.5$ and $k=0$. Under these conditions of the calculation, it is seen that $\Delta$ changes by about $0.3^{\circ}$ and $\Psi$ by $0.001^{\circ}$ for $1 \AA$ variation of the film thickness. Considering that a properly aligned ellipsometer with high-quality optics is capable of precision of about $0.01-0.02^{\circ}$ in $\Delta$ and $\Psi$, sensitivity approaching $0.01 \mathrm{~nm}$ or sub-monolayer sensitivity is achievable with the determination of $\Delta$. It is important to note that this sensitivity is valid on the average of the measurement spot (Table 2).

These results also show that the $\Delta$ parameter is the most sensitive parameter to small changes, since it varies 2.976 for $10 \AA$, against 0.015 for $\Psi$, as shown in Fig. 3. From Fig. 3 it can also be inferred that 
Table 1 Calculated $\Psi$ and $\Delta$ parameters for a c-Si substrate (with $n=3.8819$ and $k=0.019$ at $633 \mathrm{~nm}$ ) coated with a transparent film with $n=1.5$ and $k=0$ as a function of film thickness $<1 \mathrm{~nm}$

\begin{tabular}{llc}
\hline$\Delta\left(^{\circ}\right)$ & $\Psi\left({ }^{\circ}\right)$ & Film thickness $(\AA)$ \\
\hline 179.195 & 10.567 & 0 \\
178.897 & 10.568 & 1 \\
178.599 & 10.568 & 2 \\
178.302 & 10.569 & 3 \\
178.004 & 10.571 & 4 \\
177.706 & 10.572 & 5 \\
177.409 & 10.573 & 6 \\
177.111 & 10.575 & 7 \\
176.814 & 10.577 & 8 \\
176.516 & 10.579 & 9 \\
176.219 & 10.582 & 10 \\
\hline
\end{tabular}

spectroscopic ellipsometry measuring one couple of $(\Psi, \Delta)$ at each wavelength allows an improvement in accuracy on nanometric films with respect to singlewavelength ellipsometry. In the case of a substrate covered by a layer (as schematized in the bottom panel of Fig. 1), $\Psi$ and $\Delta$ are a function of various variables, i.e., $(\Psi, \Delta)=\mathrm{f}\left(\varepsilon_{\mathrm{amb}}, \varepsilon_{\mathrm{film}}, \varepsilon_{\mathrm{subs}}, d, \lambda, \phi_{0}\right)$, where $\lambda$ is the wavelength of light, $\phi_{0}$ is the angle of incidence, $d$ is the film thickness, and $\varepsilon_{\mathrm{amb}}, \varepsilon_{\mathrm{film}}$, and $\varepsilon_{\text {subs }}$ are the dielectric functions of the ambient, film, and substrate, respectively. $\lambda, \phi_{0} \varepsilon_{\mathrm{amb}}$, and $\varepsilon_{\text {subs }}$ are known parameters; hence, from a spectroscopic measurement of $(\Psi, \Delta)$ at each wavelength, two parameters, typically the thickness and the refractive index, can be derived with accuracy.

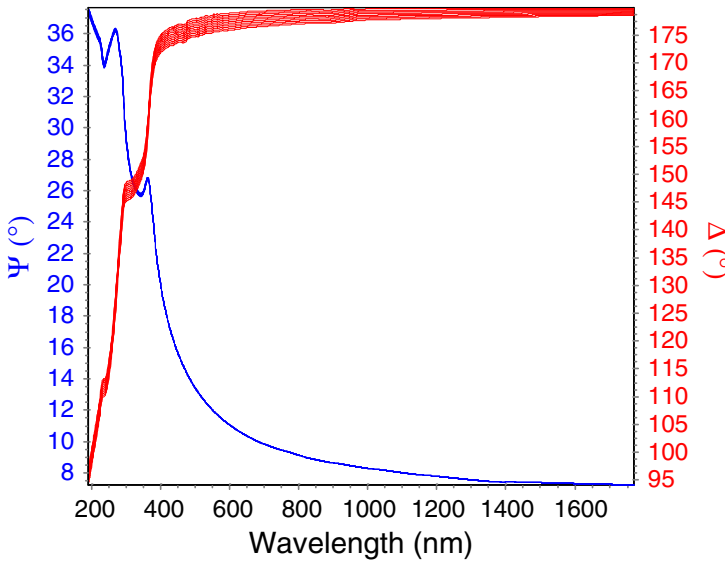

Fig. 3 Calculated $\Psi, \Delta$ results for a c-Si substrate (with $n=3.8819$ and $k=0.019$ at $633 \mathrm{~nm}$ ) coated with a transparent film with $n=1.5$ and $k=0$. Film thickness has been changed from 0 to $1 \mathrm{~nm}$. $\Delta$ changes by about $0.3^{\circ}$ and $\Psi$ by $0.001^{\circ}$ for $1 \AA$ variation of the film thickness

In order to illustrate this point, 50 measurements of native oxide made at the same spot were fitted at one wavelength of $\lambda=633 \mathrm{~nm}$, and on the whole spectral range, to determine the thickness. It can be observed that the standard deviation increases for measurements performed at only one wavelength. When the layer becomes very thin, or in case of measurements of very thin interfaces or films with a low index contrast, the technique provides the most important sensitivity in the FUV wavelength range. The example considered in Table 3 shows the variation of $(\Psi, \Delta)$ over the spectral range 0.65$6.5 \mathrm{eV}$ for a glass substrate covered with an $\mathrm{SiO}_{2}$ layer varying from 0 to $100 \AA$, by steps of $10 \AA$.

Table 2 Results for 50 measurements of native oxide on c-Si substrate by fitting full spectrum data and single-wavelength data at one wavelength of $\lambda=633 \mathrm{~nm}$ to determine thickness

\begin{tabular}{|c|c|c|c|c|c|}
\hline & Average & Maximum & Minimum & Std. Dev. & Uniformity \\
\hline \multicolumn{6}{|c|}{ Fit on the whole spectral range: $0.59-6.5 \mathrm{eV}$} \\
\hline Thickness (£) & 21.292 & 21.358 & 21.213 & 0.031 & 0.003 \\
\hline AoI & 69.927 & 69.931 & 69.922 & 0.002 & 0.000 \\
\hline$\chi^{2}$ & 0.099329 & 0.108394 & 0.089933 & 0.004176 & 0.093081 \\
\hline Iteration count & 4.7 & 5.0 & 3.0 & 0.5 & 0.3 \\
\hline \multicolumn{6}{|l|}{ Fit at $633 \mathrm{~nm}$} \\
\hline L1 thickness $(\AA)$ & 20.491 & 20.500 & 20.159 & 0.047 & 0.008 \\
\hline AoI & 69.940 & 69.954 & 69.936 & 0.007 & 0.000 \\
\hline$\chi^{2}$ & 0.000001 & 0.000001 & 0.000000 & 0.000000 & 1.000000 \\
\hline Iteration count & 2.0 & 3.0 & 2.0 & 0.1 & 0.2 \\
\hline
\end{tabular}


Table $3(\Psi, \Delta)$ values taken at $633 \mathrm{~nm}$ and at $190 \mathrm{~nm}$ for 0 and $100 \AA$ $\mathrm{SiO}_{2}$ layer on a glass substrate

\begin{tabular}{lclcl}
\hline $\mathrm{SiO}_{2} /$ glass substrate & $\Psi$ at $190 \mathrm{~nm}$ & $\Delta$ at $190 \mathrm{~nm}$ & $\Psi$ at $633 \mathrm{~nm}$ & $\Delta$ at $633 \mathrm{~nm}$ \\
\hline $0 \AA$ & 17.782 & 0.038 & 20.340 & 0.001 \\
$100 \AA$ & 18.204 & 3.233 & 20.370 & 0.861 \\
$\delta\left(\Psi_{100 \AA}-\Psi_{0} \AA\right)$ & 0.422 & 3.195 & 0.03 & 0.86 \\
$\delta\left(\Delta_{100 \AA}-\Delta_{0} \AA\right.$ & & & & \\
\hline
\end{tabular}

Table 3 compares the $(\Psi, \Delta)$ values taken at 633 and $190 \mathrm{~nm}$ for 0 and $100 \AA \mathrm{SiO}_{2}$ layer. From these results, it is inferred that the phase information $(\Delta)$ makes the measurement very sensitive with a stronger effect in the FUV. This application also shows the importance of accurate $\Delta$ measurements around $0^{\circ}$, as shown in Fig. 4, which are provided by only certain types of ellipsometer, including phase modulation, rotating compensator, or rotating polarizer/analyzer with additional retarder.

Subnanometer resolution of in situ ellipsometry for real-time monitoring of thickness evolution and film growth

In addition to accuracy and reliability, real-time measurement of thickness for nanometric films during processing is another important issue. With thin film deposition becoming increasingly critical in the production of advanced electronic and optical devices, scientists and engineers working in this area are looking for in situ, real-time, structure-specific

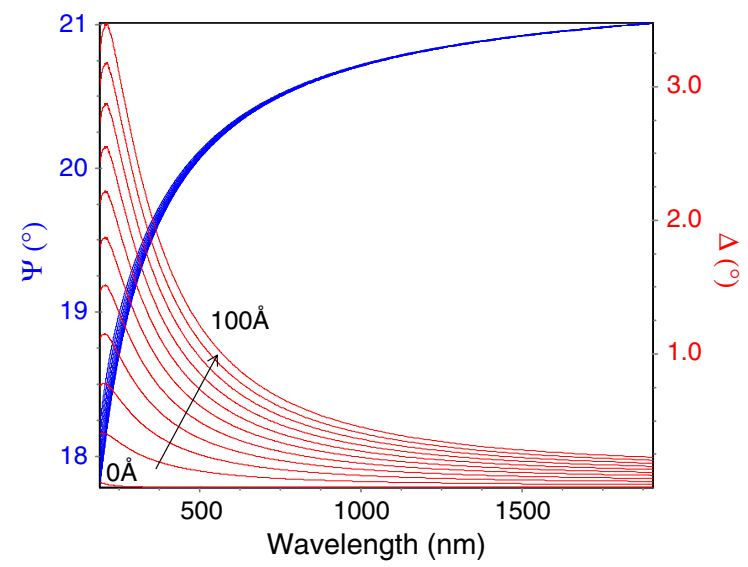

Fig. 4 Calculated variation of $(\Psi, \Delta)$ over the spectral range $0.65-6.5 \mathrm{eV}$ for a glass substrate covered with a $\mathrm{SiO}_{2}$ layer varying from 0 to $100 \AA$ by step of $10 \AA$ (see also Table 3) analytical tools for characterizing phenomena occurring at surfaces and interfaces during thin film growth. Also, in nanoscale manufacturing systems, open issues include in situ monitoring, measurement, and control of the manufacturing process. In situ monitoring parameters are indispensable not only for thin film fabrication but especially for nanostructures. Thickness and refractive index (which also relate to density and nanostructure of materials) are often the most important parameters in achieving the reproducibility necessary for technological exploitation of physical phenomena dependent on film nanostructure. Thickness is perhaps one of the most important parameters in thin film deposition, as most of the properties of a thin film (e.g., resistivity, hardness) depend on its thickness. Therefore, the control of film thickness is essential for the exploitation of physical phenomena arising from thin layers.

Recently, SE has evolved rapidly into a sensitive probe for determining the dynamics of the surface treatment and material growth because it can be deployed under the adverse conditions (including plasmas and high temperatures) used in contemporary technologies. The modern SE hardware can supply high-quality spectra within milliseconds, allowing a reasonable coverage of relatively fast processes and fast dynamics. Whenever in situ data are understood, the measurements can be used not only to monitor technological processes, but also to control them.

In order to provide an example of the real-time capabilities of ellipsometry for nanometric thickness monitoring, herein the in situ SE diagnostics of the growth of a nanometric carbon film on a metallic substrate (our substrate was a TiCN compound deposited on steel) is discussed and the key issues indicated. The specific situation discussed is the initial stage of covering the substrate by a hard carbon film, which plays a decisive role in the functionality of the product, influenced by the film quality and adhesion to the substrate. The evolution 
of the film thickness during growth is of primary importance; however, it cannot be determined without knowing the optical response functions of all the materials seen by the probing light. Therefore, a simple Drude-Lorentz model (Drude 1900; Ordal et al. 1983) of the dielectric functions has been used to represent the response functions of metals by a small number of parameters. These parameters have to be determined from the SE data, together with the film thickness. The simplest situation occurs before the beginning of the film growth, as the measured spectra can be converted easily into the dielectric function (complex permittivity) of the bare substrate. The complex reflectance ratio of the p- and spolarized light waves, $\rho=R_{\mathrm{p}} / R_{\mathrm{s}}$, plotted in Fig. 5 by solid lines, results in the spectra of Fig. 6. The response can be easily understood in terms of the free charge carriers (Drude) and interband transitions (Lorentz bands) in TiCN. Note that the data were taken at an elevated temperature, and are not accessible by ex situ measurements also due to instantaneous oxidation.

Given the sound description of the starting situation, the growth of the carbon film can be followed. A
Fig. 5 Complex reflectance ratio measured in situ on bare $\mathrm{TiCN}$ substrate (solid lines) and after depositing a carbon layer of $5.1 \mathrm{~nm}$ thickness
Fig. 6 Real, $\left\langle\varepsilon_{1}\right\rangle$, and imaginary, $\left\langle\varepsilon_{2}\right\rangle$, parts of the pseudodielectric function of the TiCN substrate, calculated from the measured data of Fig. 5 (symbols) and the best-fit approximation by DrudeLorentz lineshapes (lines)
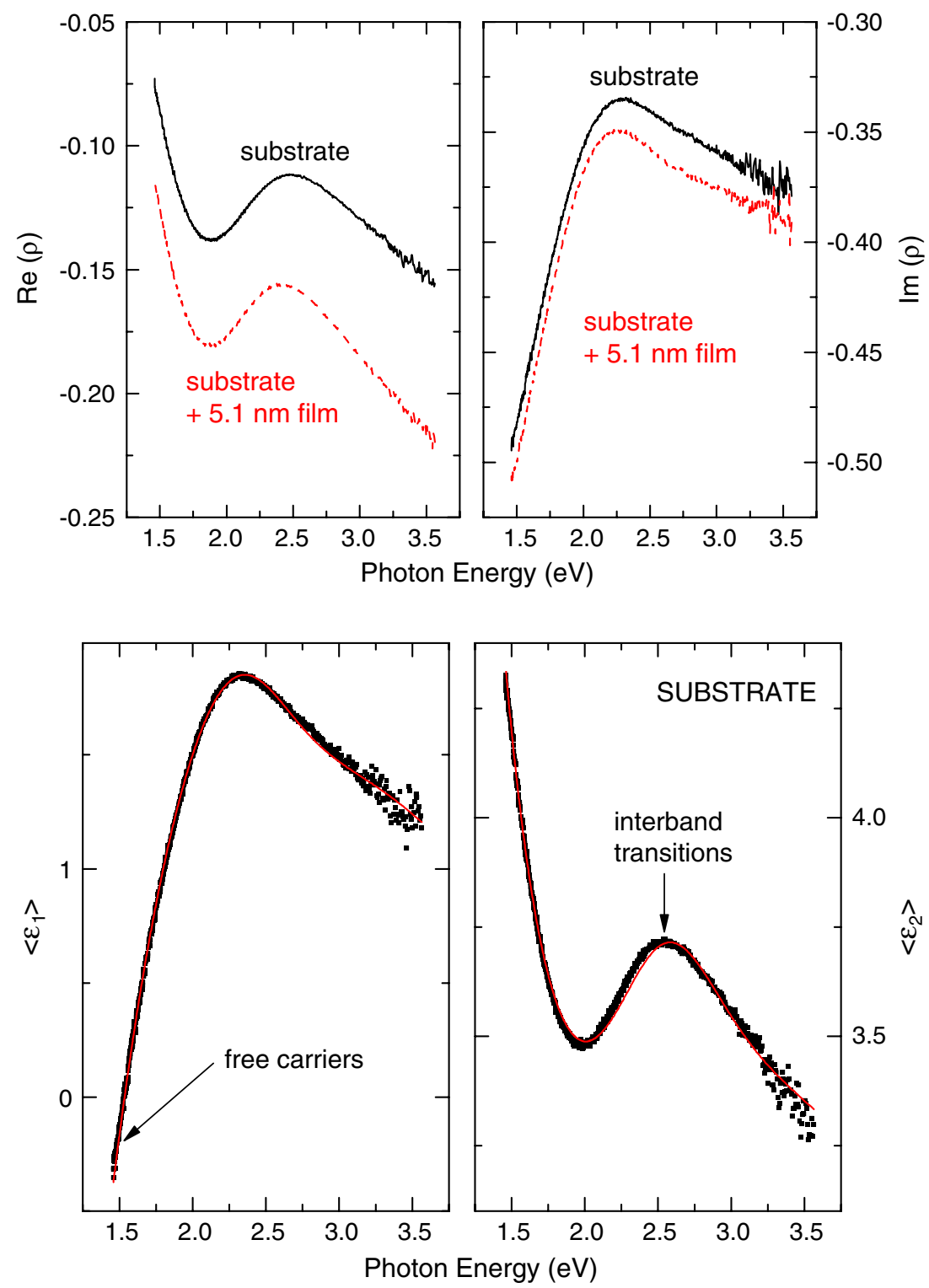
single spectrum, which turned out to be taken at the moment the total film thickness was about $5 \mathrm{~nm}$ (see Fig. 5), has been selected from a series of several hundreds. The most important effect of the presence of the film is a pronounced vertical shift of the spectra, far beyond the noise level. This indicates a spectrally flat response of the film material, which was also apparent in the very broad Lorentzian lineshapes resulting from our analysis. Further, the prevailing noise level was of the order of 0.001 in both the real and imaginary parts of $\rho$, except for the lowest and highest photon energies. As seen in Fig. 5, the ultraviolet part is noisier due to the low intensity of the probing light. The measured series of in situ spectra reveal a fairly simple temporal evolution of thickness. Growth started at a rather low rate until a thickness of about $12 \mathrm{~nm}$ has been reached, followed by a faster growth, as shown in Fig. 7. The detailed description of the properties of an evidently inhomogeneous film turned out to be rather complex. The value of static permittivity (extrapolated from the optical data) has been found to be a convenient integral characteristic of the film. It varies rather significantly in the growth direction, as seen in the right panel of Fig. 7. In any case, the variations of the optical response of the inhomogeneous film could be retrieved on the subnanometer scale.

An obvious question arising in this context, as already pointed out in the previous paragraph, is: how important is the spectroscopic version of ellipsometry with respect to single-wavelength laser source ellipsometry? Answering this question requires a deeper insight into the information content of the measured data, with the following important considerations:

(i) The spectral dependencies of the optical parameters of involved materials are very helpful in identifying the physics and chemistry of the film growth. For example, the carbon deposition is expected to start with a partial coverage of the substrate by nanometer-sized islands of rather poor compactness. Consequently, the optical absorption is located mainly in the ultraviolet; it shifts toward visible range during the later stages of deposition, due to the better tetrahedral bonding of the growing material. This behavior is indeed observed via a pronounced redshift of the spectral weight of the retrieved optical functions (Humlíček 2008).

(ii) Even if only the temporal evolution of the film thickness $d$ was important, it would not be possible to retrieve it from the data without an adequate knowledge of the optical constants. In fact, the propagation of light through the film is governed essentially by the value of the "phase," $n(d / \lambda)$, where $n$ is the refractive index and $\lambda$ the vacuum wavelength of light. Thus, $n$ and $d$ are heavily correlated: a shift of either of them from the true value results in an opposite shift of the other, keeping the product $n d$
Fig. 7 Time-dependence of the film thickness (left panel) and the static dielectric constant (right panel) retrieved from in situ SE data

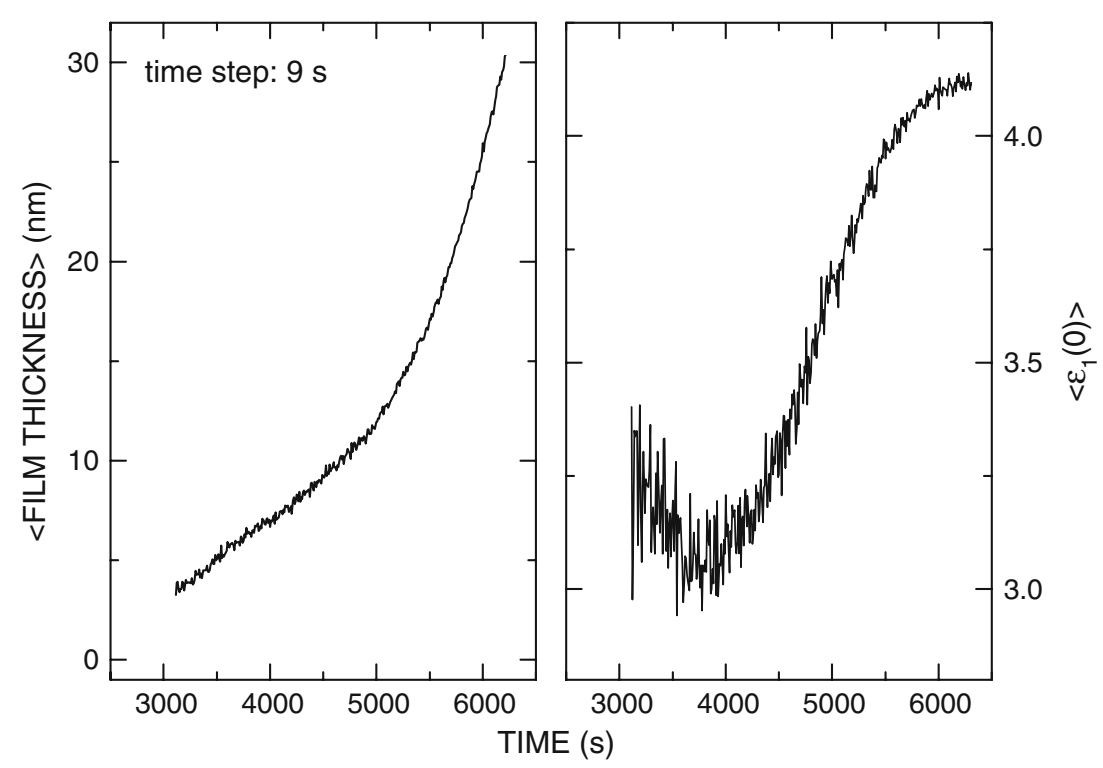


constant. When a spectral range is covered, multiple values of the ratio $d / \lambda$ enter the game, and the correlation is reduced.

The second point is illustrated in Fig. 8, where the spectral range used in the calculations (of both thickness and parametrized optical response) has been deliberately reduced to the width of $\Delta E$, narrower than the actually measured interval from 1.459 to $3.564 \mathrm{eV}$. Since the photon energies are distributed unevenly within these limits, the median differs from the mean value; the near-infrared data points have been more numerous than the nearultraviolet ones. In other words, only parts of the measured data have been used, within progressively narrower spectral windows, for smaller values of $\Delta E$. Obviously, in the limit of $\Delta E$ approaching zero, we are approaching the single-wavelength measurements performed at the photon energy, $E_{\mathrm{m}}$. A clear increase of the uncertainty with the narrowing of the spectral range covered can be seen. Note the high level of precision with respect to thickness when using the fixed optical response of the film material, quantified by $\delta_{0} d$, which is also indicated in Fig. 8 . Besides, it can also be determined which part of the spectrum is "more important" in determining the wanted parameters. The calculations have been repeated for spectral ranges centered on a lower photon energy,

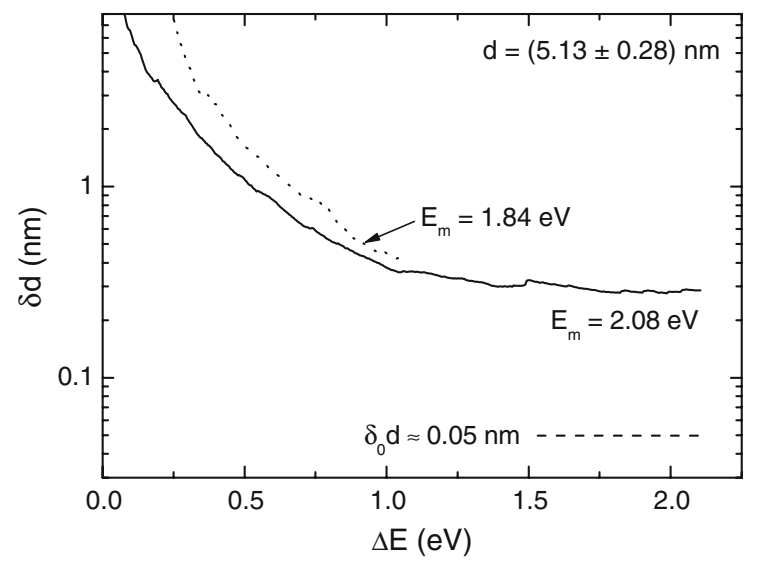

Fig. 8 Error estimates of the film thickness retrieved from the ellipsometric data of Fig. 5. The number of data points used in the fitting has been limited to cover the spectral range of the width $\Delta E$ with the median value $E_{\mathrm{m}}$ of the photon energies. The dashed line shows the statistical error of the thickness, $\delta_{0} d$, using the assumption of a known optical response of the film material and the results have been plotted as a dotted line in Fig. 8. For the same $\Delta E$, the uncertainty increased, showing the importance of the data at high photon energies, in spite of the higher noise level (see Figs. 5, 6). Thus, a proper analysis can be instrumental in specifying the conditions of meeting the targets of in situ measurements.

The example described shows that real-time diagnostics using SE is becoming a reality. Contemporary ellipsometric setups supply high-quality data in a short time, and viable calculations provide useful information for monitoring and controlling technological processes at subnanometer length scales.

\section{What can ellipsometry do for nanoparticles, nanocrystals, and nanowires?}

The interaction of light with nanometer-sized structures is at the core of nano-optics. In this section, we discuss changes of the dielectric functions of materials due to size effects and show how polarization based techniques, i.e., spectroscopic ellipsometry, reflectance anisotropy spectroscopy, and polarimetry can provide information on various nanostructures.

Resonances originating from electromagnetic and quantum mechanical effects: plasmonic metal nanoparticles

The study of optical phenomena related to the electromagnetic response of metal nanoparticles of various geometries has been recently termed plasmonics or nanoplasmonics (Maier 2007). This rapidly growing field of nanoscience is mostly concerned with the control of optical radiation on the subwavelength scale. The dynamics of a free electron gas in a finite, nanosized geometry is characterized by distinct modes known as SPR. These resonances are accompanied by enhanced electromagnetic fields. The surface charge density oscillations associated with surface plasmons at the interface between a metal and a dielectric can give rise to strongly enhanced optical near-fields which are spatially confined near the metal surface. Similarly, if the electron gas is confined in three dimensions, as in the case of a small subwavelength-scale particle, the overall displacement of the electrons with respect to the positively charged lattice leads to a restoring force, which in turn gives rise to 
specific particle-plasmon resonances, depending on the size and geometry of the particle.

Most of the studies have focused on colloidal solutions of noble Au (Kooij et al. 2002) and Ag nanoparticles whose SPR and optical properties have been measured by transmission techniques. Their optical response is analyzed by different computational methodologies, which allow the exact or approximate calculation of the optical response of nanoparticles. Among them are the Mie theory (Kreibig and Vollmer 1995), successful in explaining the coupling of isolated spherical particles embedded in an isotropic medium with the external field in the quasistatic regime; the effective medium theories, such as the Maxwell-Garnett (1904), which includes effects of particle-particle interaction as well as of the matrix materials and of the nonspherical particle shape (Maxwell-Garnett 1904); and the Rigorous Coupled-Wave Analysis (RCWA) (Moharam and Gaylord 1981; Moharam et al. 1995) provided the dielectric function is given.

One of the problems in simulating the optical response of any system is the choice of the dielectric function for the nanoparticles. The problem of determining the dielectric function at the nanoscale and using it as input in the model is not simple, since quantum-size effects also alter the dielectric function of metal nanoparticles (Kreibig and Vollmer 1995), i.e., the dielectric function is size-dependent $\varepsilon(\lambda, R)$ (where $R$ is the radius of the nanoparticles). Most of the time this size-dependence is neglected and bulk metal dielectric functions are erroneously entered in the model, causing failure in the simulation of either the spectral position or the width and amplitude of the SPR absorption band. With respect to this issue, analysis of spectroscopic ellipsometry data by using as input geometrical data of nanoparticles can be helpful to derive the nanosize-dependence of metal dielectric functions.

Even more complex is the case of nanoparticles supported on a substrate, which are surrounded by two different media (i.e., the substrate and the ambient), and whose interaction with light strongly depends on the light polarization. Their optical response upon interaction with light yields resonances with different spectral positions corresponding to plasmon modes polarized perpendicular (out-of-plane) and parallel (in-plane) to the substrate (Flores-Camacho et al. 2008) as schematized in Fig. 9. For normal incidence, or s-polarization of light, only surface plasmon oscillations parallel to the plane of the film will be excited, while the p-polarized light leads to excitation of oscillations along both axes of the spheroidal nanoparticles (Bohren and Huffman 1983). Therefore, Fig. 9 shows that two plasmon resonances are
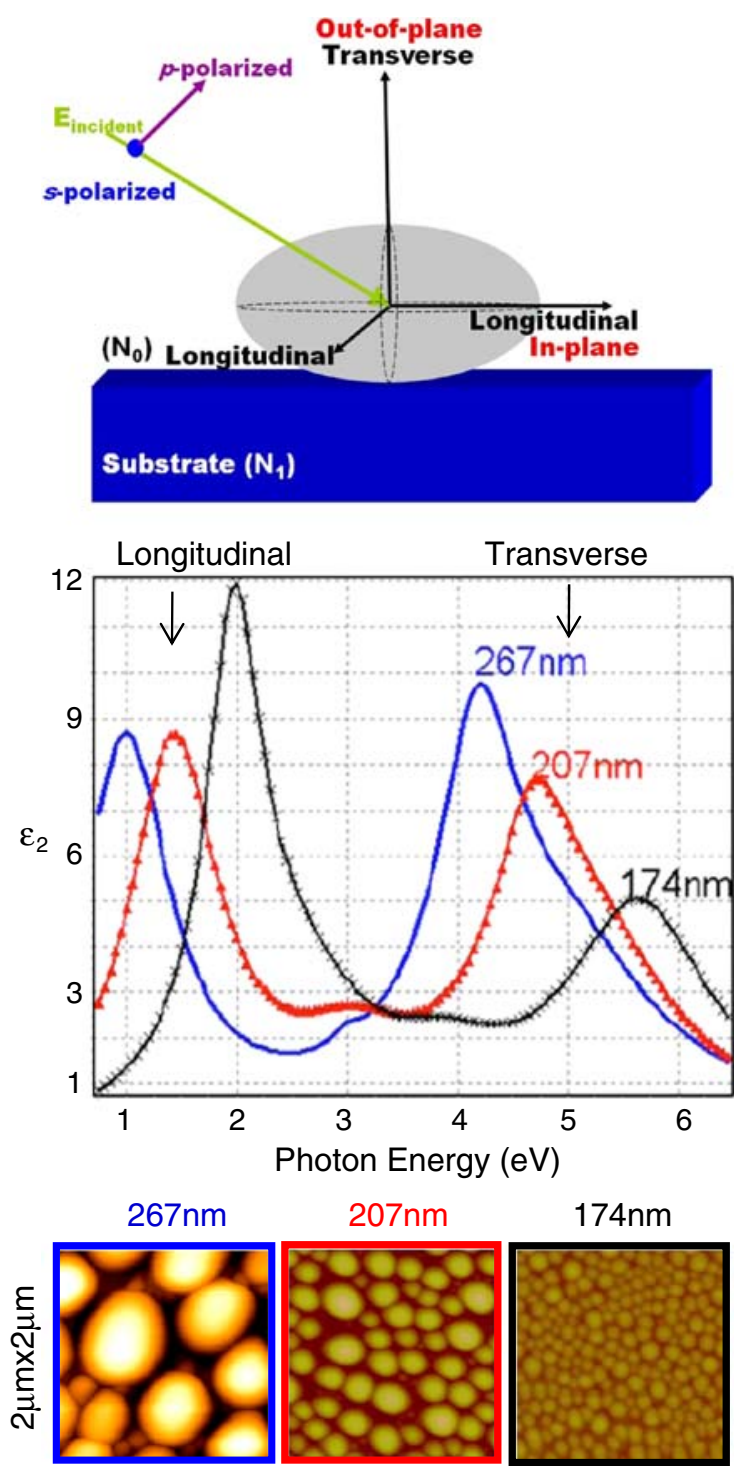

Fig. 9 Scheme of spheroidal nanoparticles supported on a substrate yielding the longitudinal and the transverse SPR modes upon interaction with polarized light. The spectra of the imaginary part of the dielectric function, $\varepsilon_{2}$, determined by ellipsometry for gallium nanoparticles of increasing size from 174 to $267 \mathrm{~nm}$ supported on a $\alpha-\mathrm{Al}_{2} \mathrm{O}_{3}$ substrate showing the two SPR modes are also shown together with the corresponding AFM images 
observed by ellipsometry, which employs oblique polarized light, for spheroidal nanoparticles of gallium deposited on a sapphire substrate. The figure also shows that both the longitudinal and the transverse SPR modes of gallium nanoparticles redshift with the increase of particle size (Wu et al. 2007). Conversely, by transmission measurements, only the longitudinal mode is probed.

Another interesting capability for the technological exploitation of nanoparticles in electronic devices, photonics, catalysis, and biochips is the possibility to monitor and control, in real time, the self-assembly and corresponding optical properties of the nanoparticles, and accurately tailor/predict the behaviors of optical devices by a simple and nondestructive method such as ellipsometry. Figure 10 shows an example of the temporal evolution of the pseudodielectric function of the $\mathrm{Ga}$ nanoparticles during their deposition on a $\mathrm{GaN}$ semiconductor substrate. A distinct resonance emerges just as the Ga nucleation begins, which results from incident photons coupling into plasmon modes of the small Ga nanoparticles. The plasmon peaks continuously redshift as the deposition time increases, which correlates to an increasing average particle size as a posteriori correlated with AFM images. Indeed, after an initial calibration of metal amount with nanoparticle size as

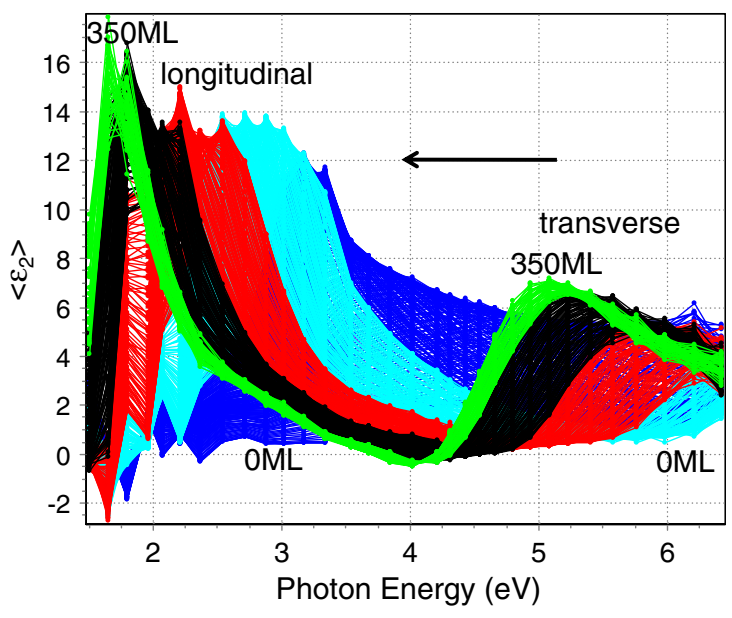

Fig. 10 Real-time spectra of $\left\langle\varepsilon_{2}\right\rangle$ recorded during deposition of Ga nanoparticles on a GaN semiconductor substrate. Spectra are shown every $1 \mathrm{~s}$ of deposition from 0 to $350 \mathrm{~mL}$ of $\mathrm{Ga}$. The spectra continuously redshift with increasing Ga amount. Both the longitudinal and transverse SPR modes are visible determined by AFM, the real-time ellipsometry data provide a means by which the formation of nanoparticles can be monitored and their functional property of plasmon resonance tuned to a specific photon energy.

Anisotropy in the free electron response of conductive atomic nanowires: contactless measure of conductivity of 2D nanostructures

Atomic scale metallic nanowires are attracting considerable attention as they show interesting fundamental effects such as Peierls instabilities, charge density waves (CDW), and spin charge separation (Himpsel et al. 2001). The nanowires of the Si(111)$(4 \times 1)$-In surface may also be considered as the smallest known conductive wires. Herein it is shown that it becomes feasible to use RAS as an optical contactless probe of conductance anisotropies in metallic nanostructures, using the case of indium nanowires on $\mathrm{Si}(111)$-the $\mathrm{Si}(111)$ : $\mathrm{In}-(4 \times 1)$ reconstruction. Such nanowires can be only 4 atoms wide and still show metallic properties. For such quasi-onedimensional systems, the measure of conductivity and its anisotropy with conventional contact-based techniques might present problems related to the characteristics of contacts. The measure is based on the fact that RAS measures the difference in the reflectance of light polarized along the two orthogonal axes $x$ and $y$ in the sample surface plane (see also Fig. 1, bottom panel), normalized to the mean reflectance

$\frac{\Delta R}{R}=2 \frac{R_{x}-R_{y}}{R_{x}+R_{y}}$.

If the complex Fresnel reflection coefficient, $\tilde{r}$, can be measured, then

$\frac{\Delta \tilde{r}}{\tilde{r}}=2 \frac{\tilde{r}_{x}-\tilde{r}_{y}}{\tilde{r}_{x}+\tilde{r}_{y}}$.

As the anisotropy is normally small, $\Delta R / R \approx$ $2 \operatorname{Re}(\Delta \tilde{r} / \tilde{r})$. Both $\Delta R / R$ and $\operatorname{Re}(\Delta \tilde{r} / \tilde{r})$ have been termed RAS signals in the literature (Aspnes 1982), but here only the latter will be used. For a three-layer system comprising an isotropic bulk with dielectric function $\tilde{\varepsilon}_{\mathrm{b}}$, an effective anisotropic layer of nanowires with dielectric function $\left(\tilde{\varepsilon}_{x}, \tilde{\varepsilon}_{y}\right)$, and air as the ambient layer (see sketch in Fig. 1), the RAS signal can be expressed as 
$\frac{\Delta \tilde{r}}{\tilde{r}}=\frac{4 \pi i d}{\lambda} \frac{\tilde{\varepsilon}_{x}-\tilde{\varepsilon}_{y}}{\tilde{\varepsilon}_{\mathrm{b}}-1}$

where $d$ is the effective thickness of the anisotropic nanowires layer. The quantity $d\left(\tilde{\varepsilon}_{x}-\tilde{\varepsilon}_{y}\right)$ is called the surface dielectric anisotropy (SDA) and is a robust quantity. Since the dielectric function is related to the optical conductivity, $\tilde{\sigma}$, by (McIntyre and Aspnes 1971)

$\tilde{\varepsilon}=1-i \tilde{\sigma} / \varepsilon_{0} \omega$

the RAS signal can be described in terms of a conductivity anisotropy $\Delta \tilde{\sigma}=\tilde{\sigma}_{x}-\tilde{\sigma}_{y}$, yielding to

$\frac{\Delta \tilde{r}}{\tilde{r}}=\frac{2 d}{\varepsilon_{0} c} \frac{\Delta \sigma}{\tilde{\varepsilon}_{\mathrm{b}}-1}$.

Therefore, the conductivity can be derived from reflectance anisotropy spectra of a 1D metallic system. The RAS spectra allow $d \Delta \tilde{\sigma}$ to be determined, analogously to the SDA. This is particularly useful, as $d \Delta \tilde{\sigma}$ is the anisotropy in the sheet conductance determined by conventional 4-point electrical measurements, assuming that the underlying bulk does not contribute significantly. Fitting the infrared RAS using the anisotropic Drude model allows $\Delta \tilde{\sigma}$ to be determined at zero frequency, thus giving an estimate of the DC conductivity. Such a calculation corresponds to an extrapolation of a measured AC conductivity to $\omega=0\left(\Delta \tilde{\sigma}_{0}\right)$.

Figure 11 shows the RAS spectrum of In nanowires on $\mathrm{Si}(111)$, whose STM image is also shown in the same figure, and it is dominated by a strong surface interband transition at $1.9 \mathrm{eV}$ and by surface contributions related to Si bulk critical points $\left(E_{0}, E_{1}\right.$, and $E_{2}$ ) (Lautenschlager et al. 1987). Nevertheless, the measurements show a small but significant IR anisotropy, which is more pronounced in the SDA (Fig. 11b). Anisotropic free electron lineshapes arising from the Drude model are shown in Fig. 12. By assuming $d=1.5 \AA$, as the metallic surface state is reported to be located at the In-Si backbonds (LópezLozano et al. 2005) and the plasma frequency, $\omega_{\mathrm{p}}$ (Ordal et al. 1983), the indium surface plasmon frequency, the anisotropic conductivity is attributed to different scattering rates (Drude 1900), $\gamma_{x}$ and $\gamma_{y}$, of the free electron gas parallel and perpendicular to the indium nanowires, which were determined by fitting to the RAS data as $\gamma_{x}=1.1 \pm 0.2 \mathrm{eV}$ and $\gamma_{y}=$ $2.8 \pm 0.4 \mathrm{eV}$. The fitted $\gamma_{x}$ scattering rate compares (a)
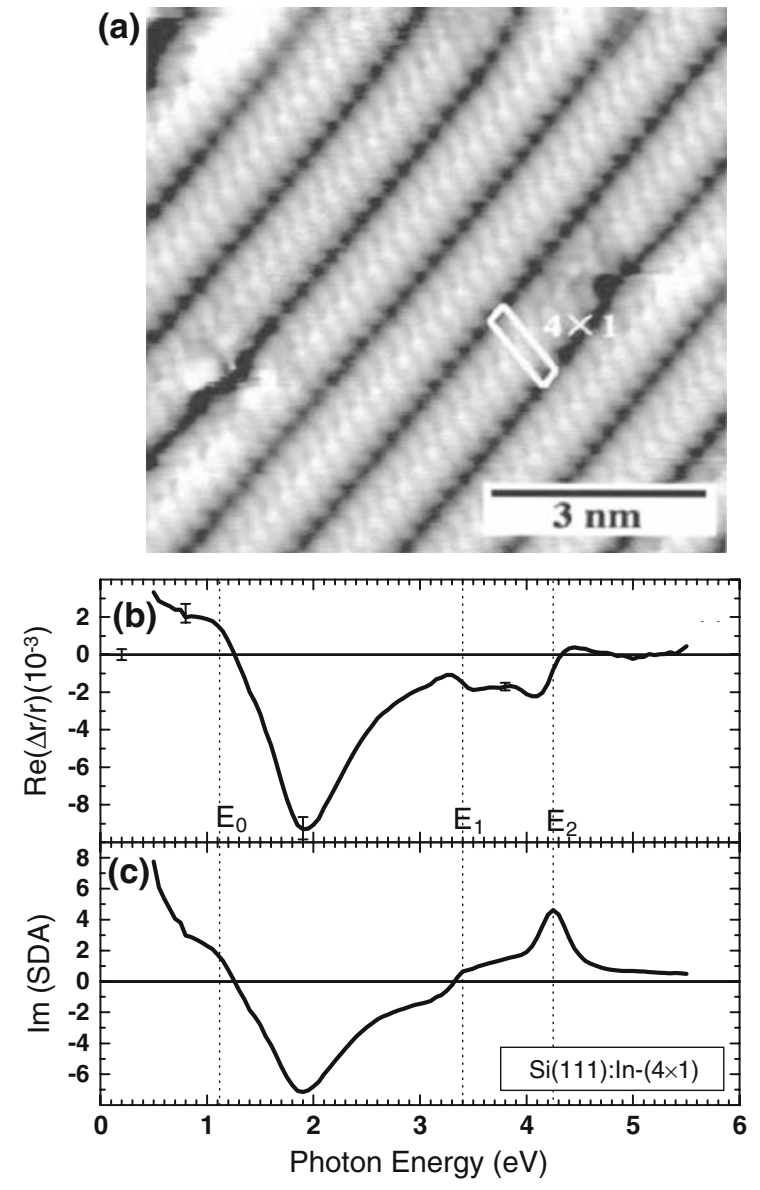

Fig. 11 a Scanning tunneling microscopy images of indium nanowires on $\mathrm{Si}(111)$ (Yeom et al. 1999). The In- $(4 \times 1)$ unit cells is indicated by a white square. b RAS and c SDA spectra of the metallic nanowires at the $\operatorname{Si}(111)$ : In- $(4 \times 1)$ surfaces. The spectra are dominated by anisotropic surface interband transitions around $1.9 \mathrm{eV}$ and $\mathrm{Si}$ critical points

well with the value given from a combined angleresolved photoemission spectroscopy and conductivity study (Kanagawa et al. 2003). In the same study, the sheet conductance of $\operatorname{Si}(111): \operatorname{In}-(4 \times 1)$ has been measured along, and perpendicular to, the chain direction, giving $\Delta \sigma=7.1 \pm 0.6 \times 10^{-4} \mathrm{~S} / \mathrm{cm}$. Assuming that the effective thickness of the DC conducting and Drude-type layers are equal, it is then possible, using the above formula $\Delta \tilde{r} / \tilde{r}=\left(2 d / \varepsilon_{0} c\right)$ $\left(\Delta \sigma / \tilde{\varepsilon}_{b}-1\right)$, to calculate the RAS signal at $\omega=0$ as $50 \pm 4 \times 10^{-3}$. This is ten times larger than the extrapolated value. Including this known DC value in the fit (dashed line in Fig. 12), the much larger DC anisotropy can be accommodated, and the scattering rates become $\gamma_{x}=0.2 \pm 0.04 \mathrm{eV}$ and $\gamma_{y}=5.3 \pm 0.8 \mathrm{eV}$. 


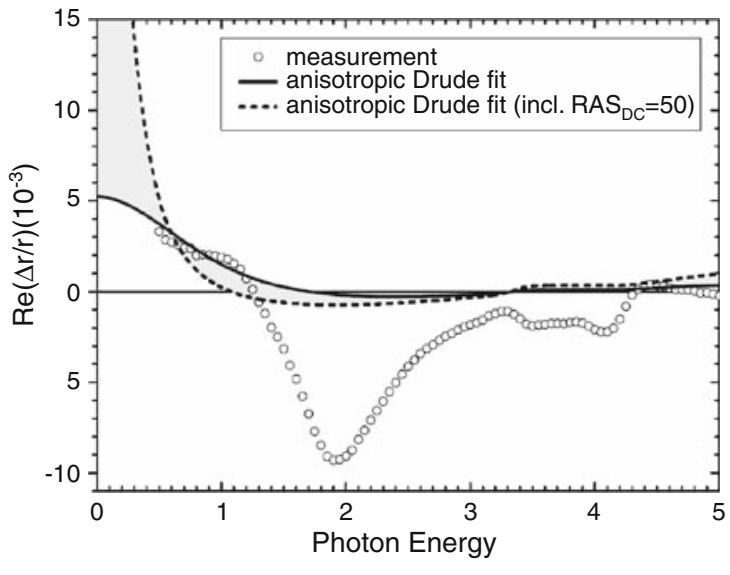

Fig. 12 Comparison of the $\operatorname{Si}(111)$ :In- $(4 \times 1)$ RAS signal with the free electron response. All model parameters except $\gamma_{x}$ and $\gamma_{y}$ were kept fixed $\left(\omega_{\mathrm{p}}=8.3 \mathrm{eV}, d=1.5 \mathrm{~nm}\right)$ and only the spectral region below $1.2 \mathrm{eV}$ and above $4.5 \mathrm{eV}$ was included in the fit. For the solid line $\left(\gamma_{x}=1 \mathrm{eV}, \gamma_{y}=2.8 \mathrm{eV}\right)$, only the RAS data were used in the fit, while for the dashed line $\left(\gamma_{x}=0.2 \mathrm{eV}, \gamma_{y}=5.8 \mathrm{eV}\right)$, the $\mathrm{DC}$ response $\left(\mathrm{RAS}_{\mathrm{DC}}=50\right)$ determined from the measured anisotropy of the sheet conductance (Yu and Cardona 1999) was included as a zero frequency datum

The shaded area indicates the variation in the free electron contributions between these two cases, showing clearly that a DC sheet conductance determined from RAS measurements made above $0.5 \mathrm{eV}$ is unreliable for this system.

The difficulty in extrapolating, successfully, the measured RAS response in this spectral range to zero frequency arises from the combination of a small infrared RAS response and stronginterband transitions. Nevertheless, from Fig. 12 it can be inferred that a small extension of the spectral range to $0.3 \mathrm{eV}$ should allow a much more accurate estimate of the DC sheet conductance anisotropy. Therefore, although metallic and semiconducting structures can be distinguished optically, for cases where stronginterband transitions are present, the spectral range has to be extended further into the IR to determine reliably the DC sheet conductance from the optical RAS response. An extension to $0.3 \mathrm{eV}$ can be achieved with a standard polarizer/PEM setup (Goletti et al. 2002). However, conceptually different setups have to be developed to move further into the IR: one approach would be the use of an FTIR ellipsometer.

A further improvement in the analysis of conductive nanowires can be achieved by overcoming the limitation of the Drude free electron model, which leads to a dielectric function involving a merely phenomenological scattering parameter. This analysis improvement can be achieved by considering screening $\left(\varepsilon_{\infty}\right)$ and by using an anisotropic effective mass of the electrons instead of the free electron mass, an approach used to describe the Drude absorption by free carriers in semiconductors ( $\mathrm{Yu}$ and Cardona 1999). This more sophisticated model could not be applied so far, because the total number of parameters becomes too large to be useful (in the absence of additional data allowing some of these to be fixed), and the spectral range is already too small for definitive fits even with the simpler model.

Quantum mechanical confinement effects in semiconductor nanocrystals: analysis of HgTe nanocrystals

The dielectric function and band structure of materials change with nanoparticle size. As the particles become smaller and smaller, the laws of quantum mechanics can become apparent in their interaction with light. In this limit, continuous scattering and absorption of light will be supplemented or replaced by resonant interactions if the photon energy hits the energy difference of the discrete internal (electronic) energy levels. In atoms, molecules, and nanoparticles, like semiconductor nanocrystals and other "quantum confined" systems, these resonances are found at specific optical frequencies.

$\mathrm{HgTe}$ nanocrystals (NCs) emit photoluminescence (PL) and electroluminescence (EL); hence, they are used to gain light in the near-infrared (Roither et al. 2005; Shopova et al. 2004). The PL and EL devices are based on quantum effects, which take place when the radius of the $\mathrm{NC}$ is reduced below the Bohr exciton radius, which is $r=40 \mathrm{~nm}$ in bulk $\mathrm{HgTe}$ (Rath 2005). Quantum confinement modifies the energy levels, and HgTe is transformed from a semimetal with a negative band gap of $\mathrm{Eg}=$ $-0.15 \mathrm{eV}$ to a semiconductor with a band gap of up to $1 \mathrm{eV}$, depending on the size of the NCs. The influence of the preparation and deposition method on the band gap and on the emission properties of the NCs has been extensively studied (Harrison et al. 1999). However, so far there are no studies on the effects of the quantum confinement on higher energy critical points for $\mathrm{HgTe}$ NCs. Ellipsometry has been 
demonstrated to be able to determine optical properties and the effects of quantum confinement in CdTe and PbSe (Babu Dayal et al. 2005; Hens et al. 2004).

Herein, we show the use of spectroscopic ellipsometry to study quantization effects in HgTe NCs, and specifically the relation between the size of the NCs and the energy shift of electronic transitions in semiconductor NCs. Transitions between bands which are closer to the Fermi energy and have a smaller carrier mass are more strongly affected by quantization. The spectroscopic ellipsometry measurements were performed in the spectral range from 1.5 to $5 \mathrm{eV}$ on $1-10$ alternate layers of polymer/HgTe nanocrystals. The NCs were prepared from colloidal solution, using an aqueous thiol-capping method (Rogach et al. 1999) with thioglycerol (TG) as stabilizer. The size of the NCs can be increased after preparation by heat treatment. The as-prepared NCs have a diameter of about $3 \mathrm{~nm}$ (hereafter denoted as "smallest"). Annealing for $10 \mathrm{~h}$ at $75^{\circ} \mathrm{C}$ results in an increase of the average size of the NCs to $\sim 10 \mathrm{~nm}$ (hereafter denoted as "largest"). NCs mono- and multilayer were self-assembled using a layer-by-layer deposition technique driven by electrostatic interaction (Decher 1997). Alternate deposition of poly(diallyl-dimethylammonium chloride) (PDDA) and TGstabilized $\mathrm{HgTe} \mathrm{NCs}$ from their aqueous solution resulted in the formation of a sequence of PDDA/ HgTe NCs bilayers.

Figure 13 shows the experimental and fit pseudodielectric function of a sample with 10 bilayers with the smallest NCs on a glass substrate. The inset shows a TEM picture of a 9-nm-large HgTe NC. Ellipsometry measurements clearly show the existence of the $E_{1}$ and $E_{1}+\Delta E_{1}$ critical points in the dielectric function of the HgTe NCs and their shift to higher energies compared to the bulk values caused by size quantization, while broadening mainly occurs due to size inhomogeneity. Interestingly, the $E_{0} \mathrm{HgTe}$ transition can only be observed in NC samples, where a band gap exists due to quantum confinement, in contrast to bulk HgTe, which is a semimetal and does not exhibit this transition. The energy position of the CPs can be determined accurately using the second derivative of the pseudodielectric function.

Figure 14 shows the second derivative of the imaginary part of the pseudodielectric function, $\left\langle\varepsilon_{2}\right\rangle$, for the largest $(10 \mathrm{~nm})$ and the smallest $(3 \mathrm{~nm}) \mathrm{NCs}$; for comparison, the second derivative of $\varepsilon_{2}$ for bulk

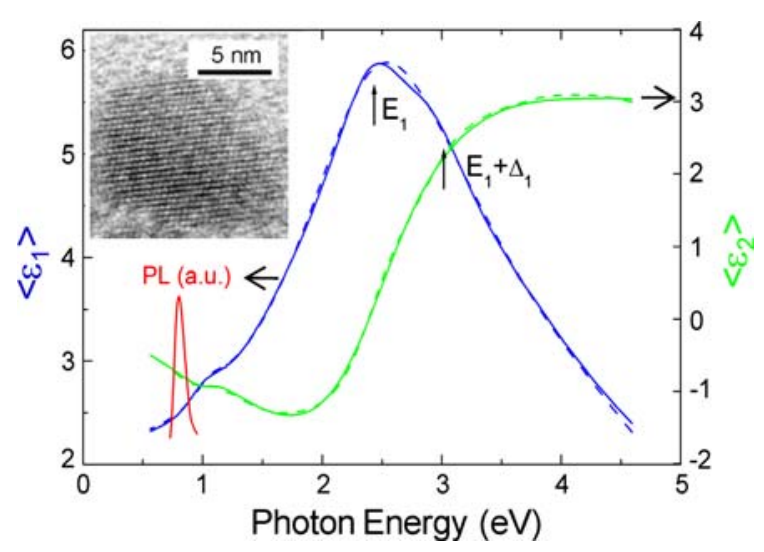

Fig. 13 SE measurement (straight line) and fit (dashed line) as well as PL measurement of a sample with 10 bilayers of HgTe NCs with a diameter of $3.5 \mathrm{~nm}$ on glass; inset: TEM picture of a NC with a diameter of $\sim 9 \mathrm{~nm}$

HgTe (Aspnes and Arwin 1984) is also shown. As can be seen, the positions of the $E_{1}$ and $E_{1}+\Delta_{1}$ CPs shift to higher energies with decreasing size, whereas there is almost no change in the position of the $E_{2}$ transition. The increase of the oscillator width due to the size distribution of the NCs can also be observed. The oscillator strength of these transitions is much smaller for the NCs than for the bulk material. The lineshape of the CPs (Rossow 1995) can be described as

$\langle\varepsilon\rangle^{\prime \prime}=f \cdot \mathrm{e}^{\mathrm{i} \phi}\left(E-E_{i}+\mathrm{i} \Gamma\right)^{-n}$

where $\varepsilon$ is the dielectric function, $f$ is the oscillator strength, $E_{i}$ is the oscillator energy, and $\Gamma$ and $\phi$ are the oscillator width and phase, respectively. In the absence of theoretical models, the exponent $n$ is set to 3 , which is used to describe excitonic effects and the phase $\phi$ is set to 0 (Rossow 1995). However, the use of different values for $n$ and $\phi$ in the fit of the measured data leads to similar results of the $\mathrm{CP}$ energies. In contrast to the NCs, the reference data of HgTe bulk material (Aspnes and Arwin 1984) were fitted with $n=2$ and $\phi=90^{\circ}$, which corresponds to the lineshape of a 2D Van Hove singularity with a saddle point in the energy band, as is the case for the $E_{1}, E_{1}+\Delta_{1}$, and $E_{2}$ transitions in HgTe. The fit of the bulk material leads to values of $2.10,2.73$, and $4.58 \mathrm{eV}$ for $E_{1}, E_{1}+\Delta_{1}$, and $E_{2}$, respectively, which is in good agreement with predicted values (Nimtz 1982). As can be seen in Fig. 14, for bulk material the strength of the $E_{2}$ transition is much smaller than for 


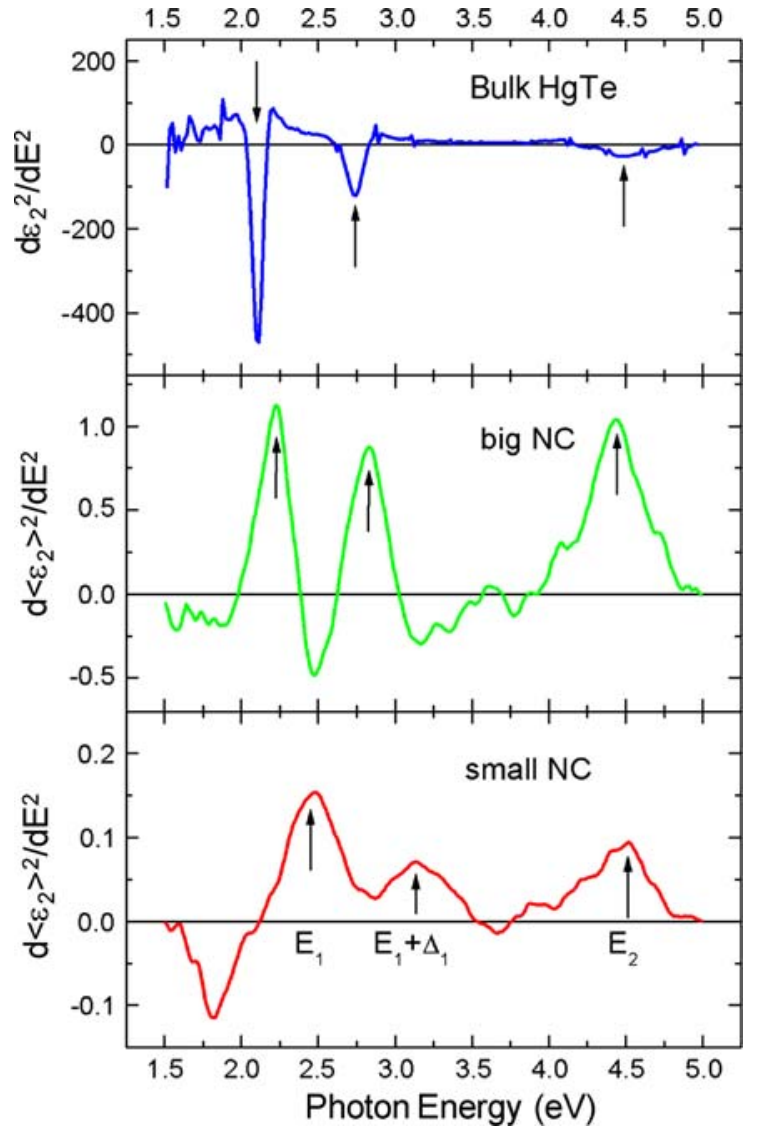

Fig. 14 Second derivative of the imaginary part of the dielectric function of bulk $\mathrm{HgTe}$ (top) and the measured pseudodielectric function of $\mathrm{HgTe} \mathrm{NCs}$ of $10.7 \mathrm{~nm}$ diameter (middle) and $3.5 \mathrm{~nm}$ diameter (bottom)

the other transitions, whereas in the NCs the relative strength of the CPs is comparable.

In contrast to the $E_{2}$ transition, the dependence of the $E_{1}$ and $E_{1}+\Delta_{1}$ transitions on their size is readily determined from the SE measurements. The energy of these transitions increases with the decreasing size of NCs, as shown in Fig. 15. For the smallest NCs with a diameter of about $3 \mathrm{~nm}$, the blueshift of the $E_{1}$ transition (heavy hole) is about $0.3 \mathrm{eV}$ and the shift of the $E_{1}+\Delta_{1}$ (light hole) transition about $0.4 \mathrm{eV}$ to higher energies compared to the bulk $\mathrm{HgTe}$ values. Even for the biggest NCs with a diameter of about $10 \mathrm{~nm}$, which is about one-fourth of the Bohr exciton radius of $\mathrm{HgTe}$, the energies of the $E_{1}$ and $E_{1}+\Delta_{1}$ transitions are well above the transition energies in bulk HgTe. In summary, it is clear that transitions between bands which are closer to the Fermi energy and have a smaller carrier mass are more strongly

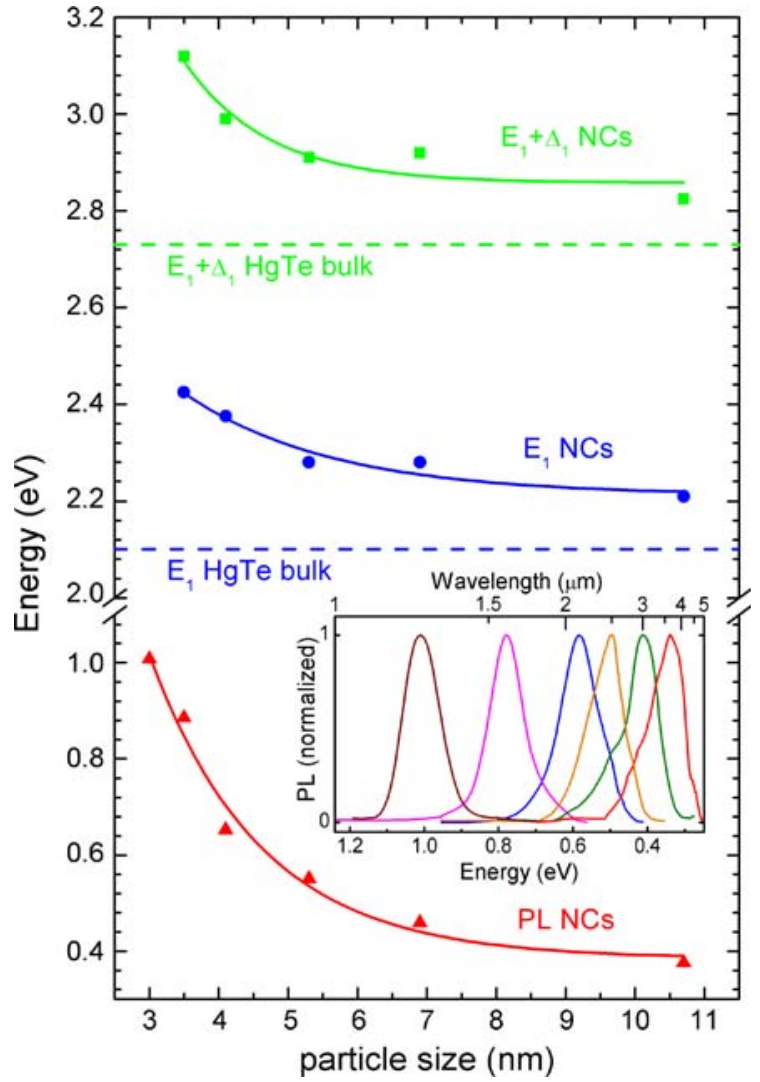

Fig. 15 Dependence of PL peak energy and higher transition energies on the size of HgTe NCs (lines are a guide to the eye)

affected by quantization, and the quantization effects can be detected and modeled exploiting ellipsometry.

Identification of defect states in nanocrystalline materials: the ellipsometric study

of nanocrystalline $\mathrm{Ce}_{0.85} \mathrm{Gd}(\mathrm{Y})_{0.15} \mathrm{O}_{2-\delta}$

When dealing with nanoparticles and nanocrystals, the role of their high surface-to-volume ratio is important. This implies that the effect of surface dangling bonds and grain boundaries, in addition to quantum effects, also contributes in making the optical response of nanocrystals different from that of the corresponding bulk materials. In the case of nanostructured oxides, O-vacancies density and localization are well known to play a determinant role in the optical and electrical properties. Defects may create localized states within the band gap, modifying the band structure profile and electronic properties which reflect in changes of the dielectric function that can be probed by ellipsometry. 
Herein, we present the example of nanocrystalline cerium oxide, $\mathrm{CeO}_{2}$, also doped with rare earth oxides such as $\mathrm{Y}$ and $\mathrm{Gd}$, to discuss how surface localized defect states can mask quantum-size effects. In particular, it is shown that ellipsometry can also be sensitive to effects of oxygen vacancies on the optical functions. In fact, nanostructured $\mathrm{CeO}_{2}$ is reported to exhibit a lower fundamental gap than crystalline $\mathrm{CeO}_{2}$, although the quantum-size effects are expected to induce a blueshift of the band gap (Kim et al. 2002; Bolotov et al. 1999), as also seen in the previous paragraph. It has already been reported that nanostructured $\mathrm{CeO}_{2}$ mainly consists of $\mathrm{CeO}_{2}$ nanocrystals or nanorods, with a considerable concentration of trivalent $\mathrm{Ce}^{3+}$ distributed at the outermost surface of the nanocrystals or nanorods (Losurdo 2004). The $\mathrm{Ce}^{3+}$ content increases with decreasing grain size, eliminating the results of the quantum-size effect and causing the redshift of the band gap (Tsunekawa et al. 2000; Patsalas et al. 2003). The additional information provided here is that the role of oxygen vacancies and interband defects also depends on doping, and the capability of using spectroscopic ellipsometry as a method of identifying defect states is explored. The ellipsometric data are herein corroborated by Raman measurements. Indeed, the approach presented here for $\mathrm{CeO}_{2}$ is generally applicable to other oxides. In particular, there are other reports in literature about exploiting ellipsometry for identifying localized charge trapping states in $\mathrm{Si} / \mathrm{SiO}_{2} /$ high-K gate stack systems (Price et al. 2007).

The example of $\mathrm{CeO}_{2}$ has been chosen since it is one of the most reactive rare earth metal oxides and has been extensively used in various applications, including catalysis, oxygen storage capacitors, UV blockers, and ion conductors. One of the most relevant applications of ceria-based materials is in solid oxide fuel cells (SOFCs) as an electrolyte material (Lee et al. 2005). Ceria-based electrolytes offer many advantages over traditional zirconia-based electrolytes (YSZ): notably higher conductivities at lower operating temperatures and compatibility with high-performance cathode materials. Among the electrolyte materials that have been widely employed in fuel cells, nanocrystalline ceria doped with rare earth oxides such as $\mathrm{Nd}, \mathrm{Y}, \mathrm{Gd}$, and $\mathrm{Sm}$ is a promising candidate for SOFCs because it exhibits higher ionic conductivity at intermediate temperatures than the corresponding bulk counterpart. Ionic conductivity is dependent on the concentration of oxygen vacancies present in the electrolyte material, and in the nano-ceria doped with rare earth oxides, the oxygen vacancy concentration can be almost three orders of magnitude higher than in polycrystalline ceria (Patil et al. 2006). These vacancies in nanocrystalline ceria can be intrinsic, originating from the reduction of the grain size and enlargement of the surface-to-volume ratio of the sample, or they can be induced by doping ceria with trivalent ions of rare earth elements (Sin et al. 2004) when replacing of $\mathrm{Ce}^{4+}$ with divalent or trivalent cations results in the creation of oxygen vacancies and predominantly oxygen ionic conductivity.

Spectroscopic ellipsometry, corroborated by Raman scattering spectroscopy, has been used for the detection of the presence of intrinsic and introduced (extrinsic) oxygen vacancies and their distribution in the bulk as well as at the surface shell of doped $\mathrm{Ce}_{0.85} \mathrm{Gd}(\mathrm{Y})_{0.15} \mathrm{O}_{2-\delta}$ nanocrystalline powders. The $\mathrm{Ce}_{0.85} \mathrm{Gd}(\mathrm{Y})_{0.15} \mathrm{O}_{2-\delta}$ nanocrystalline powders were obtained by self-propagating room temperature synthesis using metal nitrates and sodium hydroxide (Bošković et al. 2005). The particles have an average crystallite size of about $7-13 \mathrm{~nm}$ as estimated by transmission electron microscopy (TEM) and AFM images of nanocrystalline $\mathrm{Ce}_{0.85} \mathrm{Gd}(\mathrm{Y})_{0.15} \mathrm{O}_{2-\delta}$ shown in Fig. 16 and corroborated by X-ray diffraction and Raman scattering measurements. Room-temperature Raman spectra of $\mathrm{Ce}_{0.85} \mathrm{Gd}(\mathrm{Y})_{0.15} \mathrm{O}_{2-\delta}$ nanocrystalline samples obtained with three laser excitation lines (647, 514.5, and $488 \mathrm{~nm}$ ) are presented in Fig. 17. The Raman spectra obtained in Stokes excitation give information about the surface region of the samples, while the bulk states of the nanocrystalline particles can be analyzed using longer wavelengths because of the different penetration depths of the light (Gouadec and Colomban 2007).

In fact, by changing the laser excitation, it is possible to distinguish the surface from bulk composition in both samples. In the Raman spectra of these samples, besides the $F_{2 g}$ Raman mode of fluorite structure, two additional modes appear approximately at 600 and $546(550) \mathrm{cm}^{-1}$. These modes are attributed to intrinsic and extrinsic $\mathrm{O}^{2-}$ vacancies. The $\mathrm{O}^{2-}$ vacancies originating from the nonstoichiometry of ceria nanocrystallites (intrinsic vacancies) are the defect sites near the $\mathrm{Ce}^{3+}$ ions which form $\mathrm{Ce}^{3+}-\mathrm{O}^{2-}$ vacancy complexes. Therefore, the higher Raman 

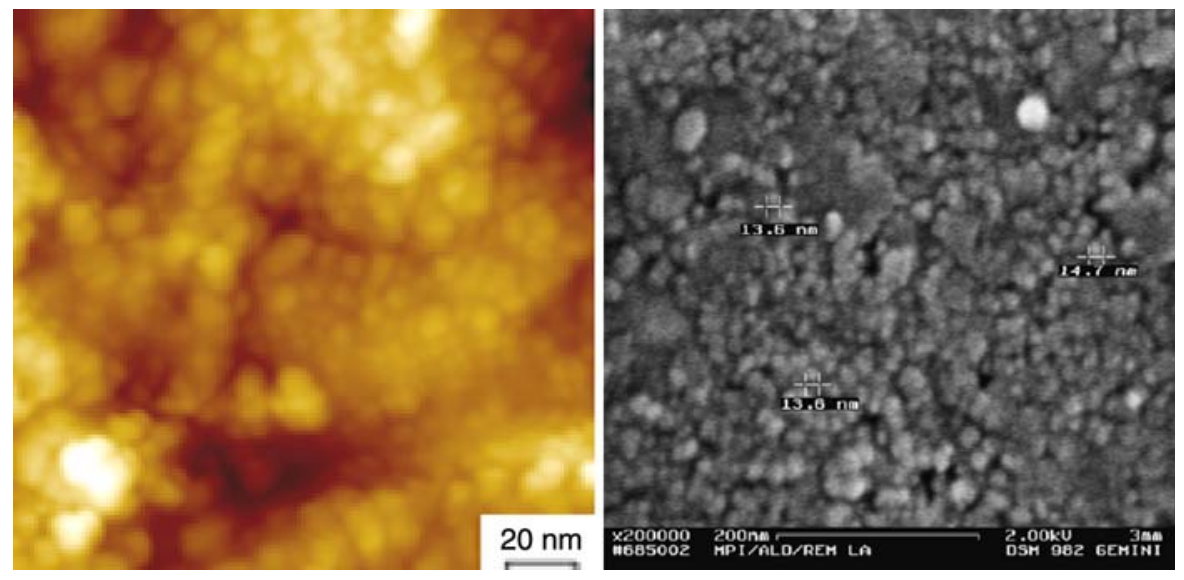

Fig. 16 AFM and TEM images of $\mathrm{Ce}_{0.85} \mathrm{Gd}(\mathrm{Y}){ }_{0.15} \mathrm{O}_{2-\delta}$ nanocrystalline samples

Fig. 17 Raman spectra of a $\mathrm{Ce}_{0.85} \mathrm{Gd}_{0.15} \mathrm{O}_{2-\delta}$ and $\mathbf{b}$ $\mathrm{Ce}_{0.85} \mathrm{Y}_{0.15} \mathrm{O}_{2-\delta}$ samples taken at room temperature with three different laser excitation lines. Change of the intensity of the extrinsic and intrinsic vacancy Raman modes with different laser excitation lines for $\mathbf{c} \mathrm{Ce}_{0.85} \mathrm{Gd}_{0.15} \mathrm{O}_{2-\delta}$ and $\mathbf{d ~} \mathrm{Ce}_{0.85} \mathrm{Y}_{0.15} \mathrm{O}_{2-\delta}$ samples
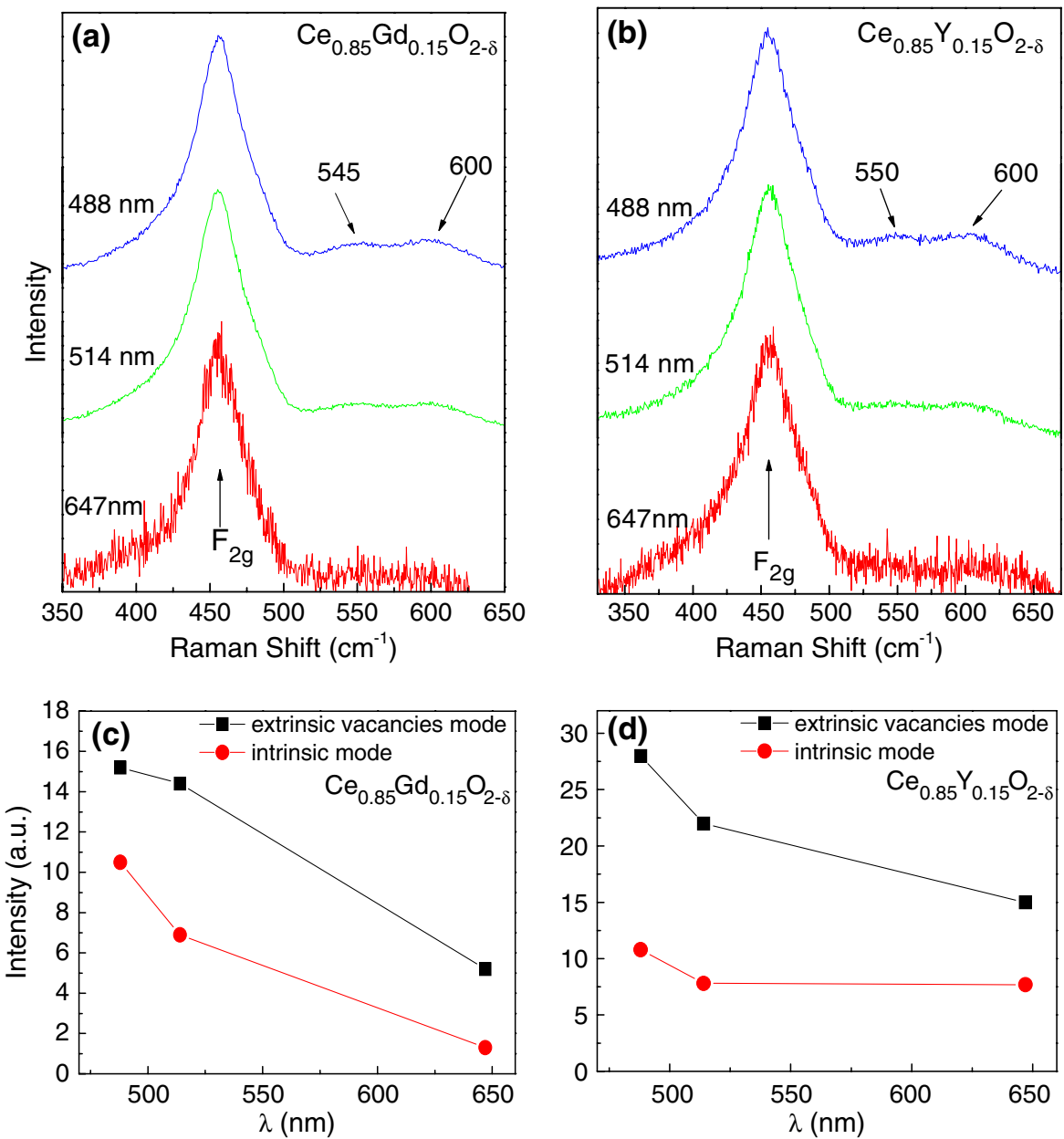

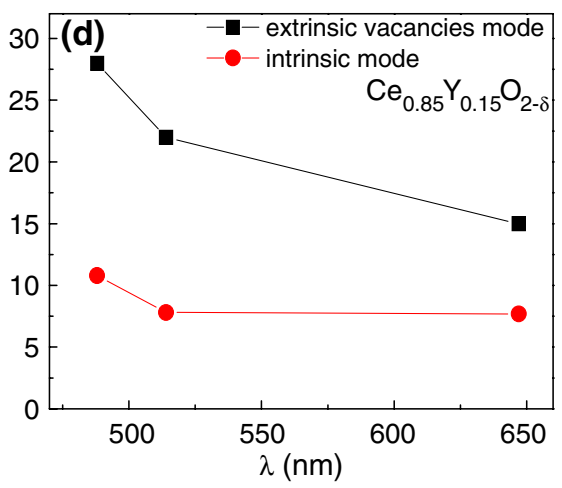

intensity of the mode at $600 \mathrm{~cm}^{-1}$ indicates the higher concentration of the $\mathrm{Ce}^{3+}$ ions in ceria lattice. The substitution of $\mathrm{Ce}^{4+}$ ions with trivalent $\mathrm{Y}^{3+}$ and $\mathrm{Gd}^{3+}$ cations results in the forming of so-called extrinsic vacancies. The existence of vacancy complexes related to the dopant ion- $\mathrm{O}^{2-}$ vacancy results in the 
appearing of new Raman mode $546(550) \mathrm{cm}^{-1}$. The higher the concentration of these complexes, the higher the Raman mode intensity (Dohčević-Mitrović et al. 2006). Deconvolution with the Lorentzian-line profile technique enables the intensity of intrinsic (extrinsic) vacancy modes to be obtained. As can be seen from Fig. 17c, d, the intensity of these modes in both Gd- and Y-doped ceria samples rises on changing the laser excitation from 647 to $488 \mathrm{~nm}$, whereas the intensity of extrinsic (intrinsic) vacancy modes is higher in the Y-doped sample. Such behavior of Raman vacancy modes suggests that in the surface layer there is a higher concentration of both types of vacancy complexes as regards the bulk part of the nanocrystals.

The modeling of the ellipsometric data for $\mathrm{Gd}(\mathrm{Y})$ doped ceria nanocrystals was done using a two-phase model (ambient/ $\mathrm{Ce}_{0.85} \mathrm{Gd}(\mathrm{Y})_{0.15} \mathrm{O}_{2-\delta}$ ). The derived spectra of the refractive index and the extinction coefficients for both samples together with a sample of pure nanocrystalline ceria are shown in Fig. 18. The two main transitions involved in the absorption of $\mathrm{CeO}_{2}$ films in the visible-UV range are from the highest occupied valence 2 p oxygen band into empty 4f states of cerium at approximately $4 \mathrm{eV}(\mathrm{O} 2 \mathrm{p} \rightarrow$ $\mathrm{Ce} 4 \mathrm{f}$ ) and from the $2 \mathrm{p}$ band into the $d$ conduction band at approximately $9 \mathrm{eV}(\mathrm{O} 2 \mathrm{p} \rightarrow \mathrm{Ce} 5 \mathrm{~d})$. As can be seen from Fig. 18, in the extinction coefficient spectra of $\mathrm{Ce}_{0.85} \mathrm{Y}_{0.15} \mathrm{O}_{2-\delta}$ sample besides the $\mathrm{O} 2 \mathrm{p} \rightarrow \mathrm{Ce} 4 \mathrm{f}$ transition (at about $\sim 4 \mathrm{eV}$ ), there are two additional peaks at $\sim 2$ and $2.5 \mathrm{eV}$ (marked with arrows in Fig. 18), whereas in the spectra of extinction coefficient of $\mathrm{Ce}_{0.85} \mathrm{Gd}_{0.15} \mathrm{O}_{2-\delta}$ sample (see Fig. 18) there is only one peak located at $\sim 2.1 \mathrm{eV}$.

These peaks are not observed in the undoped nanocrystalline $\mathrm{CeO}_{2}$, or in the spectra of the bulk counterpart. In the surface layer of the $\mathrm{CeO}_{2}$ nanocrystals, the $\mathrm{Ce}^{4+}$ ions coexist with $\mathrm{Ce}^{3+}$ ions whose concentration increases as nanocrystal size decreases. $\mathrm{Ce}^{3+}$ ions can yield characteristic localized absorption peaks below the $\mathrm{CeO}_{2}$ gap (Marabelli and Wachter 1987), so additional absorption peaks in the lowenergy tail of the extinction coefficient spectrum by doping the ceria with trivalent $\mathrm{Y}^{3+}$ and $\mathrm{Gd}^{3+}$ ions can be expected. The appearance of new peaks in the extinction coefficient spectra of doped ceria samples can be ascribed to the presence of the defect state levels inside the fundamental gap of the doped samples. In the Y-doped ceria nanocrystalline sample,

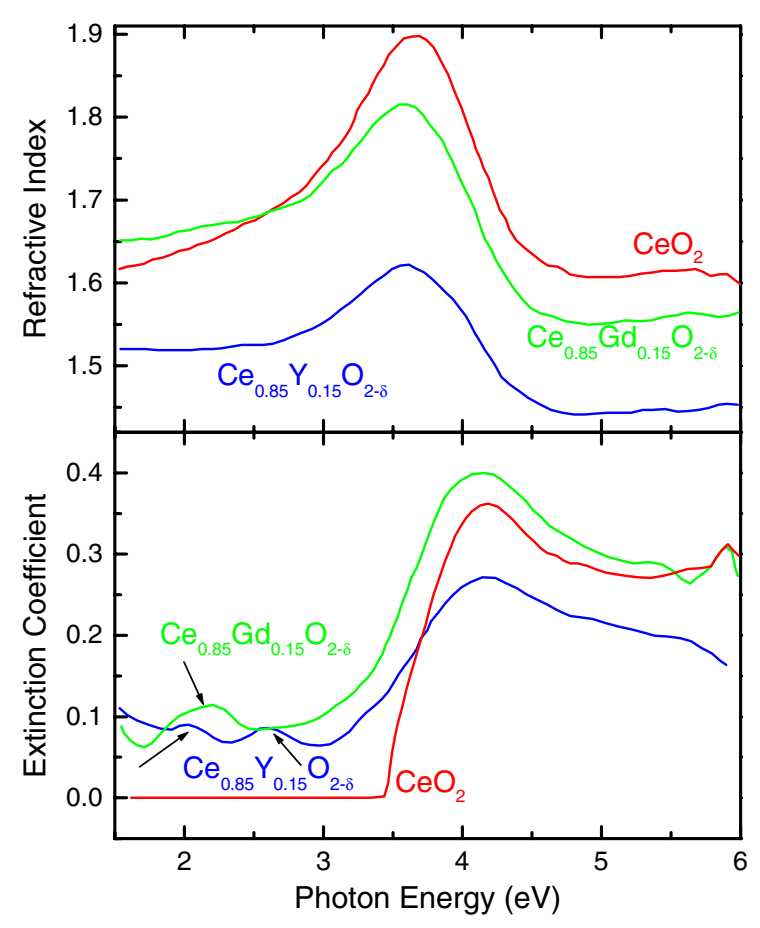

Fig. 18 Spectra of the refractive index and extinction coefficient for nanocrystalline $\mathrm{CeO}_{2}$ and $\mathrm{Ce}_{0.85} \mathrm{Gd}(\mathrm{Y})_{0.15} \mathrm{O}_{2-\delta}$ samples obtained by two-phase model analysis

a local surrounding of vacancies different from that in the Gd-doped sample (Dohčević-Mitrović et al. 2006; Deguchi et al. 2005) can be expected, yielding the formation of different structural defects. In fact, $\mathrm{Y}^{3+}$ ions tend to get together, forming a strong association with an oxygen vacancy, while Gd ions distribute randomly in ceria lattice. Ellipsometric measurements support this assumption (Fig. 18) where there is an evident difference in the extinction coefficient spectra between Y-doped (two-peak structure in the lowenergy tail) and Gd-doped ceria samples. Therefore, variable angle spectroscopic ellipsometry measurements reveal the presence of introduced defect state levels into the optical gap associated with $\mathrm{O}^{2-}$ vacancies caused by doping with trivalent $\mathrm{Gd}(\mathrm{Y})$ ions.

\section{Metrology of submicrometer gratings}

Mueller polarimetry for the metrology of submicrometer gratings

In this section we describe the application of ellipsometry and polarimetry to the economically 
very important application of process control in microelectronics.

With reference to the schematic representation of a typical MOSFET transistor (sketched on top of Table 4), the component operational characteristics, especially speed and power consumption, critically depend on its dimensions. Specifically, the electron current flowing from the source to the drain is controlled by the height of the barrier in the $p$-doped region, defined by the gate electrode potential through a thin insulating oxide layer. The characteristic length directly defined by the lithographic system is the overall spatial period, or pitch, of the structure. However, essential features like the polysilicon electrodes can be trimmed to even smaller sizes, also called CD (for Critical Dimension), by taking advantage of the nonlinearity of the insulation and etching steps. The roadmap issued by ITRS (International Technology Roadmap on Semiconductors) in terms of dimensional requirements is summarized in Table 4. Although still termed microelectronics, it is obvious from the dimensional point of view that this technology fully deserves to be called nanoelectronics. In addition to single-feature dimensional characterization, another critical issue is the measurement of overlay. Microelectronic circuits are actually made of several layers, with metal- or polysilicon-filled "contact holes" between layers and conductive lines within each layer, to properly connect the transistors sitting directly on the substrate at the bottom of the structure. To make sure the connections are operative, the successive layers must be overlaid on top of each other with an accuracy of a fraction (typically one-tenth) of the pitch, which is an increasingly challenging issue. Therefore, according to Table 5, new challenging issues appear every year for metrology, as well as for lithography.

Real space imaging techniques, including electron microscopies, both in SEM and TEM modes continue to be used to image structures, but at current scales, electron micrographs must be taken with caution, as they may be affected by artifacts. AFM is gradually emerging as a reliable standard technique, and its performance is dramatically improving with the development of engineered tips (flared, made of

Table 5 Results of the fits of the spectra shown in Fig. 20, with their estimated statistical errors $(3 \sigma)$

\begin{tabular}{lrr}
\hline Parameter & \multicolumn{1}{l}{ SE fit } & \multicolumn{1}{l}{ AFM } \\
\hline CD $(\mathrm{nm})$ & $128.2 \pm 4.8$ & $128.6 \pm 4.3$ \\
$h(\mathrm{~nm})$ & $147.9 \pm 2.2$ & $136.8 \pm 3.1$ \\
SWA $\left({ }^{\circ}\right)$ & $91.4 \pm 0.1$ & $84.3 \pm 3.3$ \\
\hline
\end{tabular}

The values of the same parameters measured by AFM are also listed for comparison

Table 4 Summary of 2007 ITRS roadmap for the current and future technological "nodes" (ITRS 2007) for a typical MOSFET transistor

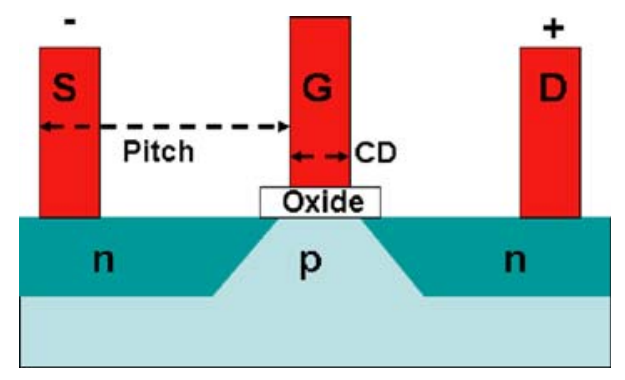

\begin{tabular}{|c|c|c|c|c|c|}
\hline Production year & 2007 & 2010 & 2013 & 2016 & 2019 \\
\hline Half pitch (nm) & 65 & 45 & 32 & 22 & 16 \\
\hline Gate electrode width (nm) & 25 & 18 & 13 & 9 & 6 \\
\hline Dense line patterns metrology accuracy $(\mathrm{nm}, 3 \sigma)$ & 1.2 & 0.84 & 0.58 & 0.42 & 0.42 \\
\hline Overlay metrology accuracy $(\mathrm{nm}, 3 \sigma)$ & 13 & 9 & 6.4 & 4.4 & 3.2 \\
\hline
\end{tabular}

The substrate is $p$-doped monocrystalline silicon, with ion-implanted $n$-doped regions

$S$ source, $G$ gate, $D$ drain 
carbon nanotubes) and scanning protocols (Foucher et al. 2008). On the other hand, the optical techniques currently used or tested for grating metrology, which include reflectometry, ellipsometry, and, more recently, Mueller polarimetry are increasingly appreciated due to their nondestructive character and their speed, which make them suitable for in-line process monitoring on a wafer-to-wafer basis.

Among these techniques, spectrally resolved classical ellipsometry is probably the most widely used metrological technique in the semiconductor industry (Sendelbach and Archie 2003; Huang and Terry 2004). The most general geometry for an optical measurement on a 1D grating is schematized in Fig. 19, with an incidence (polar) angle $\phi$ and an azimuth $\theta$. In the following, only specular reflections will be considered, even though diffraction orders other than zero may be considered for single-wavelength, angle-resolved techniques. The ellipsometric spectra are taken at incidences $\phi$ around $70^{\circ}$ (close to Si Brewster angle, a choice which is known to optimize the sensitivity to thin film parameters) and at zero azimuth. In this configuration, also called planar diffraction geometry, the symmetry of the system clearly implies that the grating cannot mix $s$ and $p$ polarizations. As a result, its Jones matrix J (Azzam and Bashara 1977) is diagonal, and takes the form (see also Section "Fundamentals")

$\mathbf{J}=\tau\left[\begin{array}{cc}\tan \Psi \exp (\mathrm{i} \Delta) & 0 \\ 0 & 1\end{array}\right]$

and can be fully determined by a classical ellipsometer in the same way as for isotropic films. The data

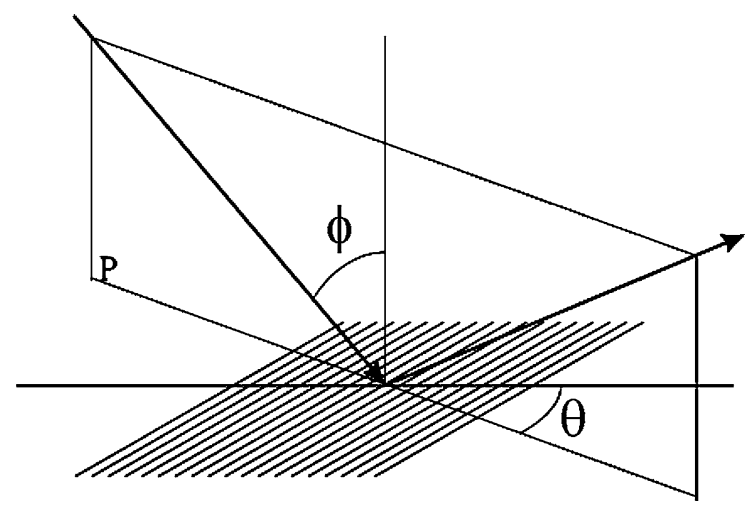

Fig. 19 General geometry of an optical measurement on a 1D grating in specular reflection. The incidence is defined by the polar angle $\phi$ and the azimuthal angle $\theta$ formed by the incidence plane $\mathrm{P}$ and the direction of the grating lines to be fitted by the multiparameter model are then the two spectra provided by the instrument (the $\Psi$ and $\Delta$ ellipsometric angles, or the equivalent quantities $\alpha=$ $-\cos 2 \Psi, \beta=\sin 2 \Psi \cos \Delta$ directly measured by rotating polarizer ellipsometers, or the $I_{\mathrm{s}}$ and $I_{\mathrm{c}}$ synchronous signals measured by phase-modulated ellipsometers and are related to $\Psi$ and $\Delta$ in a way that depends on the setting of the instrument).

An example of classical ellipsometric spectra measured on a photoresist grating on a silicon substrate is shown in Fig. 20. The grating pitch is $390 \mathrm{~nm}$, and the target profile is rectangular, with nominal values for its height $h$ and width (or CD) being 150 and $130 \mathrm{~nm}$, respectively. The measured spectra are fitted by the trapezoidal model shown in the inset of Fig. 20, with the optimal values of the parameters listed in Table 5 together with AFM data, for comparison. The parameter statistical errors, defined as $3 \sigma$, where $\sigma$ are the parameter variances

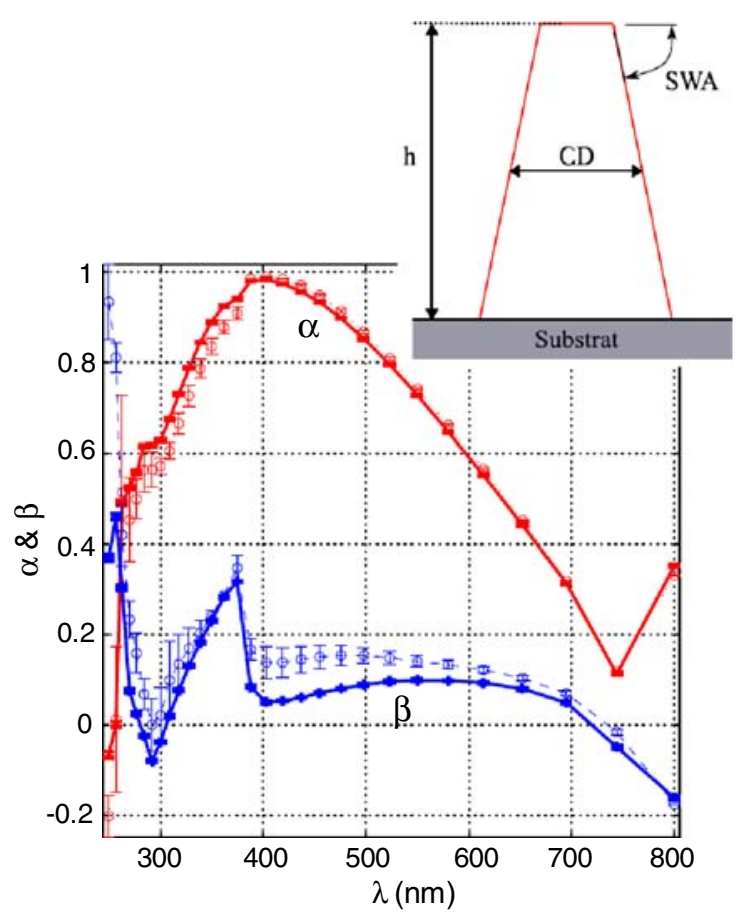

Fig. 20 Typical ellipsometric spectra taken on a photoresist grating (390 nm pitch) on $\mathrm{Si}$ substrate. $\alpha$ and $\beta$ are the ellipsometric parameters. The points are fitted quantities, with error bars deduced from the estimated statistical errors on the dimensional parameters (Quintanilha 2005). The inset shows the assumed trapezoidal profile for the photoresist grating, with the corresponding parameters ( $h$ height, $C D$ width at half height, $S W A$ sidewall angle) used to fit the measured spectra 
estimated according to well-known methods (Press et al. 1992), are found to be equal to a few nm, in spite of the relatively large standard deviation between the measured and fitted spectra. In contrast with these very encouraging results, parameter variances, in comparison with the AFM data, show significant discrepancies for both the grating height and the sidewall angle. Indeed, optical techniques, including ellipsometry, feature the main shortcomings listed below:

- They are indirect methods, relying on the solution of an inverse problem. The solution of this problem actually depends on the multiparameter model used to describe the structure. Moreover, for a given model the solution may be ambiguous, with several profiles giving equivalent fits to the measured data. It is then of paramount importance to constrain the dimensional parameters by including as many independent data as possible in the fitting procedure.

- They can be used only on periodic structures. As the chips themselves are typically not periodic (with, however, the noticeable exception of DRAM memories), the optical measurements are made on special square target gratings etched in the scribe lines between the chips, as shown schematically in Fig. 21. This characterization is therefore based on the assumption that the dimensional characteristics of the test gratings between the chips are representative of those of the chips themselves. Though this assumption is basically correct, with the continuous shrinking of

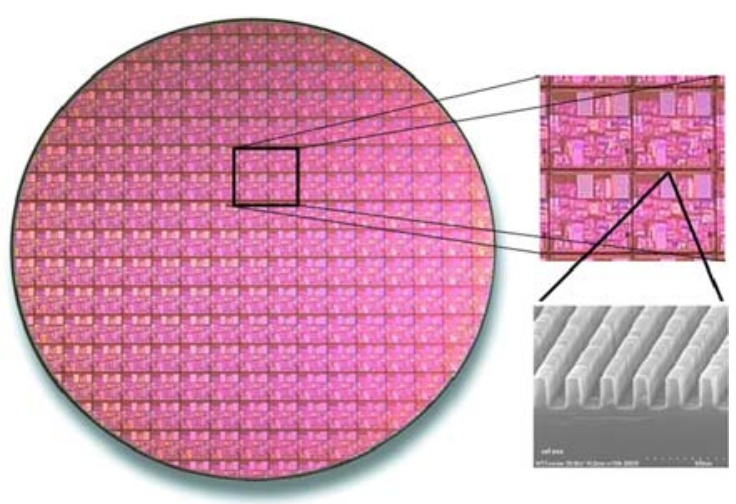

Fig. 21 Photograph of an entire wafer, with a zoom on a set of four chips separated by the scribe lines where the $50-\mu \mathrm{m}$-wide target gratings devoted to optical measurements are made the $\mathrm{CD}$, the need for measuring at least the overlay with a reasonably dense sampling inside the chips themselves is clearly emerging. Such measurements would require much smaller targets than the $50 \times 50 \mu \mathrm{m}^{2}$ gratings currently used in the scribe lines.

- Precision and accuracy, which deserve the comments below.

The presented results are quite typical, and more recent data would provide even smaller parameter variances. This is why classical ellipsometry in planar diffraction has become so popular in the semiconductor industry: the parameter variances provide a good estimate of the parameter precision, which quantifies the sensitivity of the system to small changes in dimensional parameters. So far, this sensitivity has been considered as the most crucial performance indicator for tools dedicated to process control, as these tools were essentially expected to monitor small drifts in the wafer process. Therefore, these tools (optical as well as CD-SEM) were not required to be really accurate, as it has been considered acceptable to calibrate the tools by extensive sampling and comparison with reference tools (including destructive ones like TEM) during the process development.

Now, as the wafer size (and value) is continuously increasing, such extensive, time consuming and costly characterization during the early stages of a new process is less and less acceptable. Accuracy is emerging as a major requirement for semiconductor metrology. In this respect, it is pointed out that absolute accuracy is difficult to assess in the absence of a "perfect" reference tool, which unfortunately does not exist for the current needs of microelectronics. The concept of accuracy is then replaced by that of TMU (for Total Measurement Uncertainty) (ITRS07).

In an extensive experimental investigation of these issues aimed at evaluating the readiness of optical tools to meet the requirements of the $64 \mathrm{~nm}$ technology node defined by the ITRS (Ukraintsev 2006), it was clearly shown that the parameter correlations related to the fitting procedure of optical data were the limiting factor for accuracy: if one parameter is 
changed, the quality of the fit can be restored by changing another, correlated parameter. This situation may lead to ambiguities and systematic errors when the values of the same dimensional parameter obtained by using different tools are compared. This is so, in particular, for sidewall angles of trapezoidal photoresist lines, due to the strong correlation between this parameter and the line height (Ukraintsev 2006), in good agreement with the results shown above. To address these issues, which may become increasingly severe in next generation nodes, new developments, based on spectrally or angularly resolved Mueller polarimetry, are currently underway.

\section{The Stokes-Mueller formalism}

Considering the measurements made at nonzero azimuth, the symmetry arguments (explaining why the grating does not mix $\mathrm{p}$ and s polarizations) are no longer valid; therefore, the grating Jones matrix in this more general conical diffraction geometry is no longer diagonal. Measurement of the most general nondiagonal Jones matrix is usually termed generalized ellipsometry, and it is more complex to implement than classical ellipsometry: seven real quantities (leaving apart an overall phase factor) are needed instead of two. However, generalized ellipsometry is not the most general polarimetric technique, as it assumes the sample to be nondepolarizing. Depolarization occurs whenever different polarization states add up their intensities incoherently. Such partially polarized states are no longer described by a Jones vector, but by a Stokes vector $\mathbf{S}$, defined from intensities and not field amplitudes as (Azzam and Bashara 1977)

$$
\begin{aligned}
\mathbf{S}^{\mathrm{T}} & =(I, Q, U, V) \\
& =\left(I,\left\langle I_{0}-I_{90}\right\rangle,\left\langle I_{+45}-I_{-45}\right\rangle,\left\langle I_{\mathrm{L}}-I_{\mathrm{R}}\right\rangle\right)
\end{aligned}
$$

where $I_{\alpha}\left(\alpha=0^{\circ}, 90^{\circ}, \pm 45^{\circ}\right)$ is the intensity detected through a linear polarizer oriented at an angle $\alpha$ from the p plane, and $I_{\mathrm{L}}$ and $I_{\mathrm{R}}$ are the intensities detected through circular left and right polarizers.

Upon interaction (transmission, reflection, scattering, etc.) with a sample, the Stokes vector is transformed linearly, according to $S_{\text {out }}=M S_{\text {in }}$, where the $4 \times 4$ real matrix $\mathbf{M}$ is the Mueller matrix of the considered sample. The Stokes-Mueller formalism provides the most general description of light polarization states and sample polarimetric properties. In particular, any sample, be it depolarizing or not, can be characterized by a Mueller matrix, while the converse is not true (a depolarizing sample cannot be described by a Jones matrix). For nondepolarizing samples, the Mueller matrix can be deduced from the Jones matrix by a quite simple relationship (Anderson and Barakat 1994).

\section{Application of spectroscopic Mueller polarimetry to grating metrology}

As a result, a Mueller polarimeter, when available, can advantageously replace a generalized ellipsometer. This type of instrument, operated in much the same way as a spectroscopic ellipsometer (at discrete angles and full spectral resolution; Garcia-Caurel et al. 2004), has been used in several instances to characterize gratings in conical diffraction geometries (Novikova et al. 2006, 2007; Foldyna et al. 2008). An example of the raw, spectrally resolved Mueller matrices measured on a grating at different azimuths is shown in Fig. 22. These spectra exhibit several features that are always found in this type of measurement:

- The raw data change drastically with the azimuthal angle. As the Mueller spectrum taken at each individual azimuth is already sufficient to reconstruct the profile by fitting the data with simple models, a significant assessment of the model validity is to compare the parameters obtained from the spectra taken at different azimuths.

- The Mueller matrices exhibit model-independent simple symmetries which may prove very valuable to assess the accuracy of the polarimetric measurements themselves. For instance, when the azimuth $\theta$ is changed into $-\theta$, the Mueller matrix $2 \times 2$ diagonal blocks are left unchanged, while the other elements change sign. Moreover, for symmetrical gratings, additional transposition symmetries occur at any given azimuth: $M_{i j}=M_{j i}$, except for the third line and column, for which $M_{i 3}=-M_{3 i}(i \neq 3)$. These model-independent properties (their proof is outside the scope of this review) may be of great help to decide whether the observed deviations between measurements and 

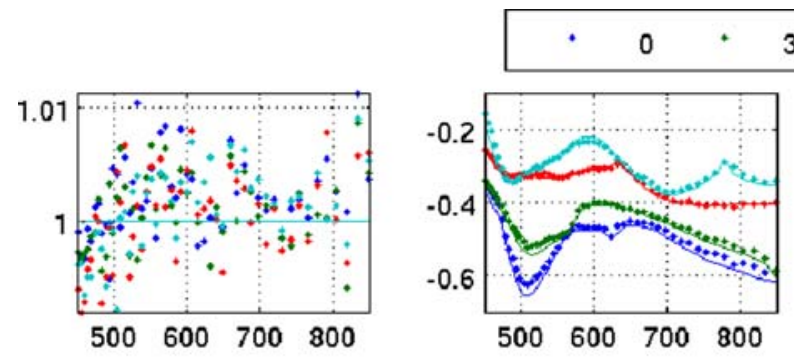

30
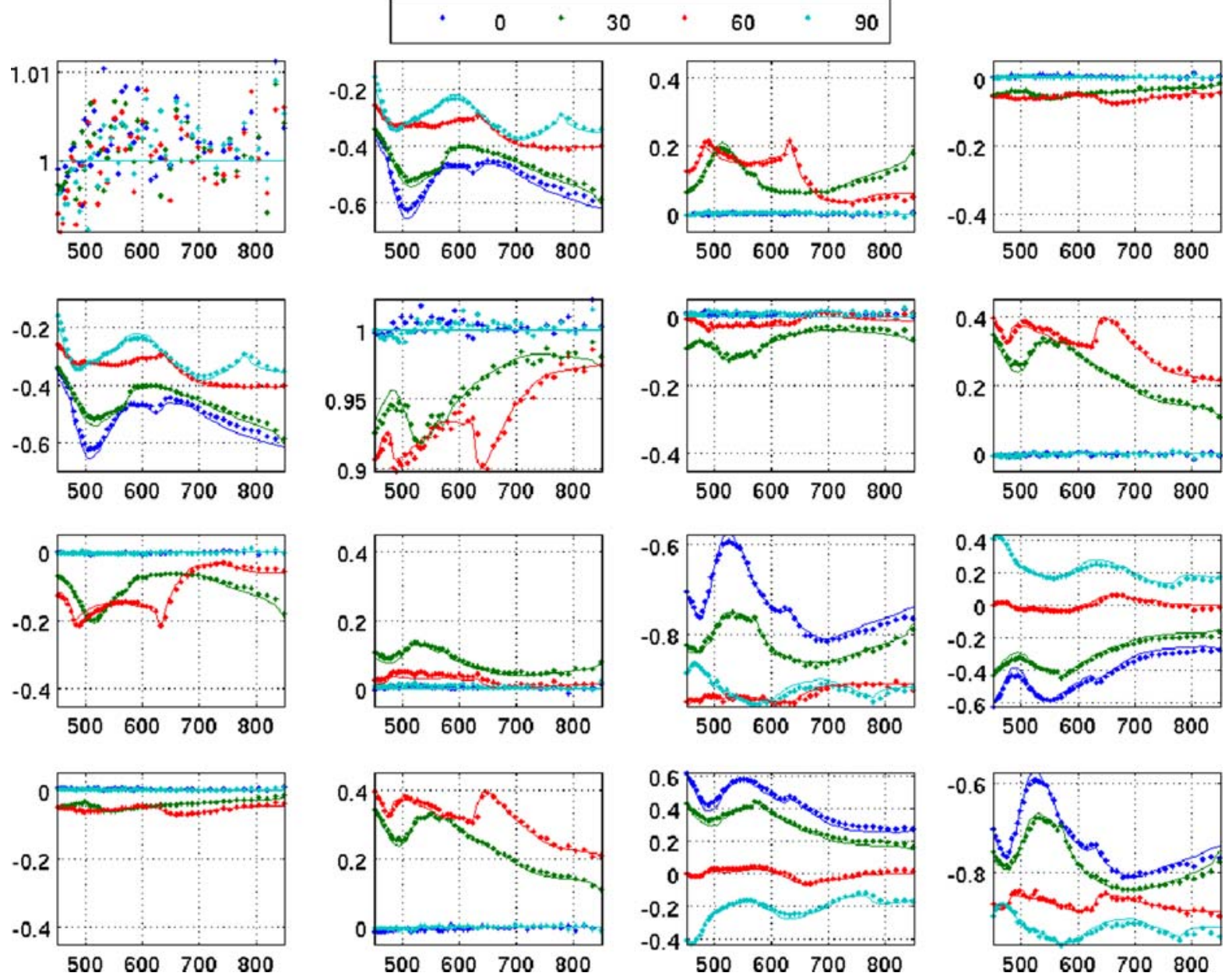

Fig. 22 Raw Mueller matrices measured on a grating etched in crystalline silicon, for $0^{\circ}, 30^{\circ}, 60^{\circ}$, and $90^{\circ}$ azimuths (solid lines) and fits by a double rectangular model (dots)

simulations are due to systematic errors in the measurements or model inadequacies.

As an example of the usefulness of these properties, it is pointed out that the spectra shown in Fig. 22 were first fitted by a trapezoidal model (De Martino et al. 2008), with a very satisfactory $\chi^{2}$ (which represent the fit quality) at each azimuth $\theta$, but with a very significant variation of the profile parameter with $\theta$ (the grating depth could vary by up to $10 \mathrm{~nm}$ ). An alternative model, consisting of two rectangular profiles on top of each other, exhibited similar values of $\chi^{2}$ at each azimuth, but with a much smaller variation of the parameters, as shown in Fig. 23. These values are in excellent agreement with those provided by state-of-the-art AFM. This example illustrates the importance of collecting as much "different" data as possible to obtain the most robust solution of the inverse diffraction problem. In this respect, Mueller polarimetry at various azimuths may prove to be a very useful technique for accurate and reliable dimensional metrology of "simple" profiles.

\section{Limitations, implementations, and future trends}

Going to the nanoscale-what are the limitations we are facing?

Going to the nanoscale, limits or better improvements that ellipsometry techniques need concern both 
Fig. 23 Variations of the $\mathrm{CD}$ and the grating depth with azimuth when the data shown in fig. $n+9$ are fitted by a double rectangular profile. The values of these same parameters taken from state-of-the-art AFM profiles are shown at the top of the figure

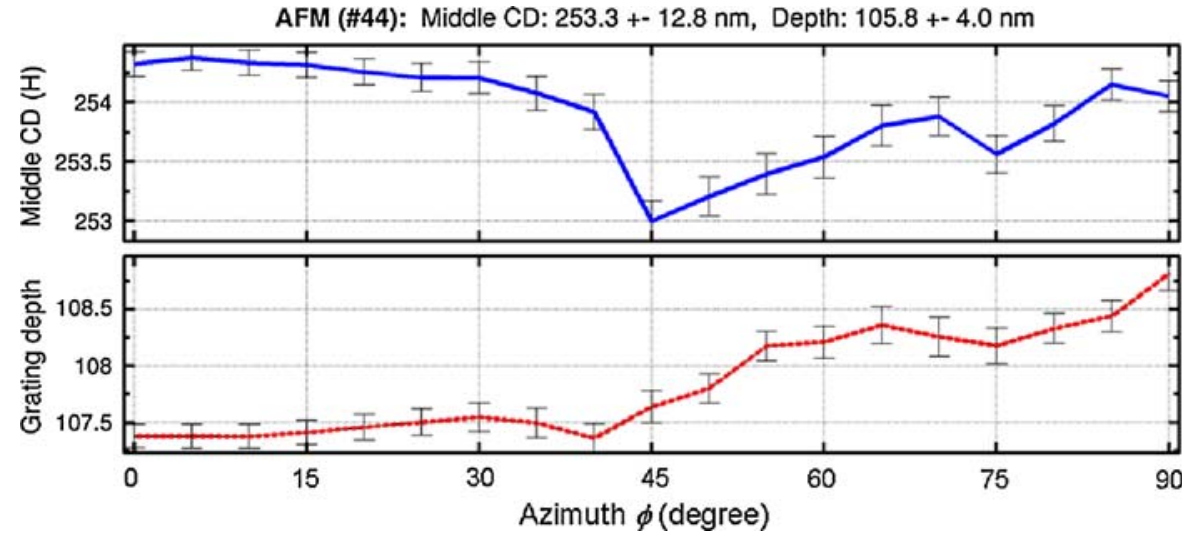

measuring and analysis. As for measurements, the main limit of this optical technique is the size of the measurement spot providing the lateral resolution of the instrument. Standard spot sizes of spectroscopic ellipsometers range from 3 to $1 \mathrm{~mm}$ in diameter, while microspot sizes are between 50 and $25 \mu \mathrm{m}$, depending on the spectral range of the measurement. A microspot is especially necessary for measurements of patterned samples or small features found in microelectronics and nanoelectronics, for display, photovoltaic, photonic, and bio devices, as discussed in Section "Metrology of Submicrometer Gratings".

The microspot is also required for accurate measurement of inhomogeneous samples exhibiting depolarization. Depolarization of the light beam can occur with nonuniform layer thickness, backside reflections from a transparent substrate, strong roughness, or a thick layer. The example below deals with the characterization of a thick, nonuniform, polymer layer. The sample has been measured with two different spot sizes, 1 and $0.1 \mathrm{~mm}$. Measurement with the smallest spot provides a better resolution of interference fringes as shown in Fig. 24. When both a small microspot and a large spectral range are required, the optical design of spectroscopic ellipsometers has to be optimized to provide the highest signal-to-noise ratio for accurate measurements.

As for analysis, limits are dictated by the sizedependence of the dielectric function, which requires standardization of nanometric and nanostructured materials and a novel dielectric function and model to describe quantum effects. As an example, the inability of actual models, theory, and effective medium approximations (EMAs) to provide a reasonable estimate for the particle size below $2 \mathrm{~nm}$ is
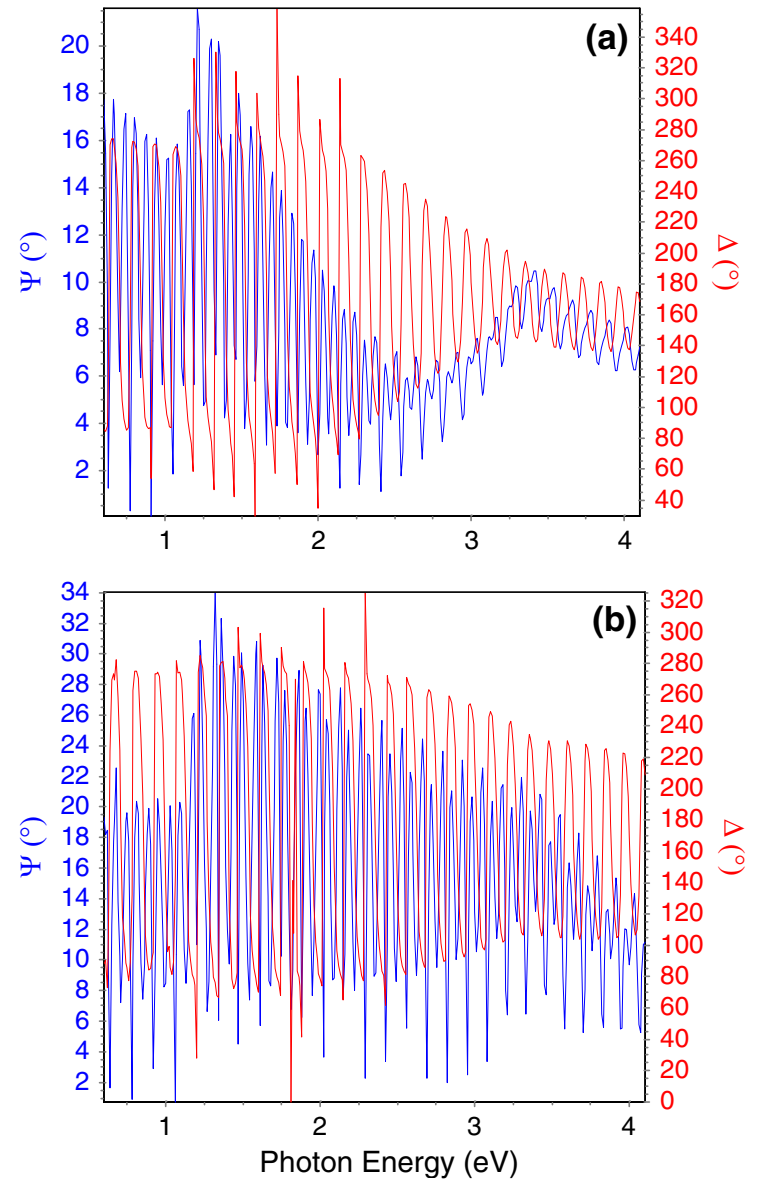

Fig. 24 Experimental data measured with a $1 \mathrm{~mm}$ spot size and $\mathbf{b} 0.1 \mathrm{~mm}$ spot size

primarily due to particle size effects influencing the band structure of the particles. It has been shown that for gold particles smaller than ca. $2 \mathrm{~nm}$, the interband transitions deviate from those in the bulk (Kreibig 
1978), therefore requiring size-dependent dielectric functions to be set for the nanoparticles and models. Furthermore, to overcome the EMA's inherent limitation due to the finite-wavelength, size, and shape effects, alternative approaches to analyze SE measurements of nanoparticles, nanorods, and nanostructures in general should be considered, like the RCWA (Moharam and Gaylord 1981; Moharam et al. 1995) as well as an efficient finite-element Green's function approach (Chang et al. 2006).

New enhancements in instrumentation and measurements

To meet the increasingly demanding need of nanometrology, a day-by-day evolution of ellipsometers is going on (Teboul 2008). The last evolution of the Auto SE spectroscopic ellipsometer (Horiba Jobin Yvon 2008) provides direct integration of a confocal visualization system enabling accurate positioning of the measurement spot. For many cases, this feature is mandatory as illustrated in Table 6. Also, this is especially a very relevant advantage for the selection and measurement of only the front reflection for thin transparent substrates of thickness $<1 \mathrm{~mm}$, such as plastic films or glass substrates found in display, optical coatings, and flexible applications. Down to a thickness of $0.4 \mathrm{~mm}$, the backside reflection can be eliminated. Furthermore, the increased complexity of samples, including depolarization phenomena and anisotropic properties, requires complete polarization measurement which is done by a polarimeter. An ellipsometer that includes a compensator or photoelastic modulator is capable of measuring up to 12 elements of the Mueller matrix, while a polarimeter provides the full 16 elements of the matrix (Horiba Jobin Yvon 2005).

The Mueller ellipsometer provides the unique combination of a classical ellipsometer + polarimeter. It uses liquid crystal modulation technology to modulate the polarization without any mechanical movement. The input head and the output head are identical and comprise a polarizer, two ferroelectric liquid crystals, and a fixed retardation plate. The light is analyzed by a spectrograph using a CCD detector and is able to deliver the complete 16 element Mueller matrix in less than $2 \mathrm{~s}$ across the spectral range 430$850 \mathrm{~nm}$. In its basic classical ellipsometer configuration, this ellipsometer is ideal for routine thin film analysis as it is very fast and very simple to operate. With the combination of polarimetric measurements, it provides advanced capabilities for biological applications, grating measurements, and the characterization of complex birefringent structures.

\section{Conclusions and challenges}

Spectroscopic ellipsometry is now a mature technique which has been successfully applied to a large variety of thin film applications. Starting 30 years ago with semiconductor research applications, spectroscopic ellipsometry has been serving the world of thin films, being used to understand material properties and processes. Nowadays, to give an idea about exploitation of ellipsometry, 300 patents related to ellipsometry are filed each year, dealing with instrumentation and more and more with applications. Driven by nanotechnology, ellipsometry is now being explored and exploited for the nondestructive and contactless characterization of nanomaterials and nanostructures.

The world of nanomaterials is continuously evolving by creating new materials with new properties. The Royal Society and Royal Academy of Engineering categorized nanomaterials in three ways: 1-dimensional (1D), e.g., thin film and layers, 2-dimensional (2D), e.g., nanotubes and nanowires, and 3-dimensional (3D), e.g., fullerenes, dendrimers, and quantum dots.

Spectroscopic ellipsometry is successfully used in the first category, that is, all applications involving thin films. The technique enables the measurement of thin layers of a few nanometers thickness, as when nanoparticles are included inside the layer, the optical properties change and need to be characterized. Regarding nanoparticle characterization, the properties of interest are typically the particle size distribution and nanoparticle density. This is a good example to illustrate the main challenge of the evolution of the technique: the identification of properties that can be characterized and the developments required to achieve this. The technique uses EMA enabling the characterization of a mixture of several materials inside a layer, and the question isshould this technique be theoretically investigated for its potential in nanoparticle characterization?

For the second and third categories, for single nanotube or fullerene characterization, the 
Table 6 Examples of samples advantaging by a visualization system

\begin{tabular}{lll}
\hline Sample type & Advantages of the MyAutoView Vision System & Examples \\
\hline A patterned sample & $\begin{array}{c}\text { Direct visualization of the measurement spot } \\
\text { on the pattern area }\end{array}$
\end{tabular}

A sample having spots

deposited on the surface

A transparent sample

A rough sample, or an inhomogeneous surface (stripe, stain)

A microstructured sample

A sample with a cylindrical or spherical shape

Sample placed inside accessories such as a liquid cell, temperature controlled stage, etc.
Direct visualization of the measurement spot on the sample to optimize measurement position

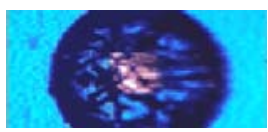

Direct selection of the front reflection only

Calculation of the degree of backside reflection for cases where the backside reflection cannot be eliminated

Direct visualization of the measurement spot on the sample to optimize measurement position

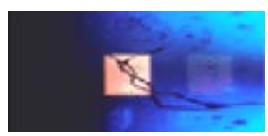

Direct visualization of the measurement spot on the sample to characterize the $0.8 \mathrm{~mm}$ line feature

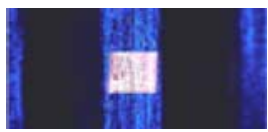

Direct visualization of the plane surface for positioning of the spot on this area. The image on the right shows that the spot is not optimally positioned, and is still on the spherical surface

Easy positioning of the measurement on a sample placed inside a liquid cell (this example)
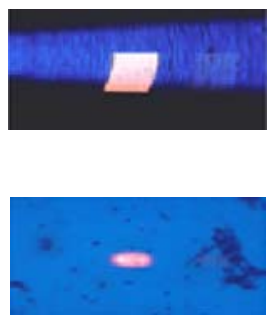

ellipsometry technique is limited by two factors: the size of the measurement spot, as the properties measured are an average of what is detected inside, and the imaging capabilities, which are compatible with the characterization of $2 \mathrm{D}$ and $3 \mathrm{D}$ features. As soon as these materials are used in films, their average properties, including thickness and optical constants, can be characterized. The recent developments of near-field ellipsometers, which demonstrate lateral resolution of $100 \mathrm{~nm}$, may be the solution for such applications. Furthermore, it has to be considered that characterization needs vary for research-oriented and industrial applications. While research requires the highest accuracy possible, industrial applications require effectiveness, reliability, speed, and cost-effectiveness in their measurement system. For 50 years, ellipsometry has continuously evolved to perfectly fit industrial requirements. Ellipsometry is used every day in labs to control the production of memories and microprocessors, flat panel and OLED displays, optical coatings, and organic lighting. Moreover, we can 
think of a bright future for spectroscopic ellipsometers as they combine accuracy, speed, and proven reliability with the huge advantage of nondestructive characterization.

The main challenge of metrology tools in industry is their integration to perfectly fit the specific process requirements. For that, the hardware design of the tool is the key. The current successful challenge is the in-line integration of ellipsometers for roll-to-roll manufacturing processes. Such processes require very fast measurements, as the speed of the film can reach several hundred meters per second, and the ellipsometer must not be sensitive to high levels of vibration. Opening new markets to ellipsometry also requires that the technique is made compatible with the materials and processes used. The technique has always been used in layered thin film stacks considered as optically homogeneous and isotropic. One can see that nowadays new materials, new nanomaterials, and substrates such as plastic, metals, textile, living materials are being employed in new applications. As the technique is based on extensive modeling, new modeling features are required. And very often, the solution is based on the respective understanding of the characterization needs of these novel applications, the theoretical developments of new functions by scientists, and the final hardware design of the metrology tool by the manufacturer.

Another consideration, from the hardware point of view, is that ellipsometers, even if they can be quite compact tools, are still not portable. Samples need to be held on the sample stage to be measured. The most successful popularization of this technique will be when the tool can be held directly in the hand of the user to measure the sample of interest without any constraints of settings, adjustments, and preparation. Despite problems that still need to be overcome when dealing with the nanoscale, spectroscopic ellipsometry provides huge advantages, such as speed and accuracy; moreover, it is nondestructive, there is no sample preparation, and it is compatible with liquid and solid samples, and absorbing and transparent substrates. This is unique for metrology instrumentation!

To conclude, education and popularization of the technique are also key challenges that the project "NanoCharm" aims to address. By facilitating ellipsometry access to scientists and industries, bridges between the product designer, the product manufacturer, the metrology tool manufacturer, and the metrology provider will be built, which will help in the design of future ellipsometers and polarimeters to characterize the upcoming functional nanomaterials.

\section{About NanoCharM}

The EU-funded NanoCharM project www.nano charm.org aims not only at promoting a greater knowledge of ellipsometry and polarimetry for the characterization of thin films and other nanomaterials amongst the research, developer, and user communities, but also at catalyzing further research and development in the technique itself. To these ends, the NanoCharM partners have established a dedicated Web site, which provides a wealth of free information, including training and demonstration videos, and are constantly seeking from the user community data on their metrology and characterization needs, in order to serve them better. The NanoCharM project partners are committed to a program of education and training in ellipsometry and polarimetry, and hold Summer Schools regularly throughout Europe, as well as offer advice and help at many conferences and exhibitions. Interested individuals are urged to visit the NanoCharM Web site, join the NanoCharM community, and contribute to a better understanding of nanoscale phenomena and how nanomaterials can be effectively characterized, using the optical techniques of ellipsometry and polarimetry.

Acknowledgment The authors acknowledge the 7th FP European Project NanoCharM (Multifunctional NanoMaterials Characterization exploiting Ellipsometry and Polarimetry) (NMP3-CA-2007-218570).

\section{References}

Anderson DGM, Barakat R (1994) Necessary and sufficient conditions for a Mueller matrix to be derivable from a Jones matrix. J Opt Soc Am A 11:2305-2319. doi:10.1364/ JOSAA.11.002305

Archer RJ (1964) In: Passaglia E, Stromberg RR, Kruger J (eds) Ellipsometry in the measurement of surfaces and thin films. National Bureau of Standards, Misc. Publ. 256, US Government Printing Office, Washington DC

Aspnes DE (1982) Local-field effects and effective-medium theory-a microscopic perspective. Am J Phys 50:704709. doi:10.1119/1.12734

Aspnes DE (2005) Real-time diagnostics for metalorganic vapor phase. Phys Status Solidi B 242:2551-2560. doi: 10.1002/pssb.200541109 
Aspnes DE, Arwin H (1984) Nondestructive analysis of native oxides and interfaces on $\mathrm{Hg}_{1-x} \mathrm{Cd}_{x}$ Te. J Vac Sci Technol A 2:600-601. doi:10.1116/1.572455

Aspnes DE, Harbison JP, Studna AA, Florez LT (1988) Application of reflectance difference spectroscopy to molecular-beam epitaxy growth of GaAs and AlAs. J Vac Sci Technol A 6:1327-1332. doi:10.1116/1.575694

Azzam RMA, Bashara NM (1977) Ellipsometry and polarized light. North-Holland, New York

Babu Dayal P, Mehla BR, Paulson PD (2005) Spin-orbit splitting and critical point energy at $\Gamma$ and $L$ points of cubic CdTe nanoparticles: effect of size and nonspherical shape. Phys Rev B 72:115413-115416. doi:10.1103/PhysRevB.72.11 5413

Beydaghyan G, Buzea C, Cui Y, Elliott C, Robbie K (2005) Ex situ ellipsometric investigation of nanocolumns inclination angle of obliquely evaporated silicon thin films. Appl Phys Lett 87:153103. doi:10.1063/1.2084329

Bohren CF, Huffman DR (1983) Absorption and scattering of light by small particles. Wiley, New York

Bolotov L, Tsutiya T, Nakamura A, Ito T, Fujiwara Y, Takeda Y (1999) Semimetal to semiconductor transition in ErP islands grown on $\mathrm{InP}(001)$ due to quantum-size effects. Phys Rev B 59:12236-12239

Bošković S, Djurović D, Dohčević-Mitrović Z, Popović ZV, Zinkevich M, Aldinger F (2005) Self-propagating room temperature synthesis of nanopowders for solid oxide fuel cells (SOFC). J Power Sources 145:237-242. doi:10.1016/ j.jpowsour.2005.01.085

Carroll JJ, Melmed AJ (1969) Ellipsometry-LEED study od adsorption of oxygen on (011) tungsten. Surf Sci 16:251264. doi:10.1016/0039-6028(69)90022-3

Chang YC, Li G, Chu H, Opsal J (2006) Efficient finite-element, Green's function approach for critical-dimension metrology of three-dimensional gratings on multilayer films. J Opt Soc Am A 23:638-645. doi:10.1364/JOSAA. 23.000638

Cobet C, Schmidtling T, Drago M, Wollschläger N, Esser N, Richter W, Feenstra RM, Kampen TU (2003) Surface termination during $\mathrm{GaN}$ growth by metalorganic vapor phase epitaxy determined by ellipsometry. J Appl Phys 94:6997-6999. doi:10.1063/1.1623630

Collins RW, Ferlauto AS, Ferreira GM, Chen C, Koh J, Koval RJ, Lee Y, Pearce JM, Wronski CR (2003) Evolution of microstructure and phase in amorphous, protocrystalline, and microcrystalline silicon studied by real time spectroscopic ellipsometry. Sol Energy Mater Sol Cells 78:143-180. doi: 10.1016/S0927-0248(02)00436-1

Cuypers PA, Corsem JW, Janssen MP, Kop JMM, Hermens WT, Hemker HC (1983) The adsorption of prothrombin to phosphatidylserine multilayers quantitated by ellipsometry. J Biol Chem 258:2426-2431

Dalacu D, Martinu L (2000) Spectroellipsometric characterization of plasma-deposited $\mathrm{Au} / \mathrm{SiO}_{2}$ nanocomposite films. J Appl Phys 87:228-235. doi:10.1063/1.371849

De Martino A, Foldyna M, Novikova T, Cattelan D, Barritault P, Licitra C, Hazart J, Foucher J, Bogeat F (2008) Comparison of spectroscopic Mueller polarimetry, standard ellipsometry and real space imaging techniques (SEM and 3D-AFM) for dimensional characterization of periodic structures. Proc SPIE 6922:69221P. doi:10.1117/12.772 721

Decher G (1997) Fuzzy nanoassemblies: toward layered polymeric multicomposites. Science 277:1232-1237. doi: 10.1126/science.277.5330.1232

Deguchi H, Yoshida H, Inagaki T, Horiuchi M (2005) EXAFS study of doped ceria using multiple data set fit. Solid State Ionics 176:1817-1825. doi:10.1016/j.ssi.2005.04.043

Dohčević-Mitrović ZD, Grujić-Brojčin M, Šćepanović M, Popović ZV, Bošković S, Matović B, Zinkevich M, Aldinger F (2006) $\mathrm{Ce}_{1-x} \mathrm{Y}(\mathrm{Nd})_{x} \mathrm{O}_{2-\delta}$ nanopowders: potential materials for intermediate temperature solid oxide fuel cells. J Phys Condens Matter 18:S2061-S2068. doi:10.1088/0953-8984/ $18 / 33 / \mathrm{S} 22$

Drude PKL (1900) On the electron theory of metals. Ann Phys 1:566-613. doi:10.1002/andp.19003060312

Flores-Camacho JM, Sun LD, Saucedo-Zeni N, Weidlinger G, Hohage M, Zeppenfeld P (2008) Optical anisotropies of metal clusters supported on a birefringent substrate. Phys Rev B 78:075416-075419. doi:10.1103/PhysRevB.78.075 416

Foldyna M, De Martino A, Cattelan D, Bogeat F, Licitra C, Foucher J, Barritault P, Hazart J (2008) Accurate dimensional characterization of periodic structures by spectroscopic Mueller polarimetry. Proc SPIE 7140:714001

Foucher J, Pargon E, Martin M, Reyne S, Dupréa C (2008) Paving the way for multiple applications for the 3D-AFM technique in the semiconductor industry. In: Metrology, inspection and process control for microlithography XXI, volume 6922 of proceedings SPIE, pp 69220F1-69220F9

Gallas B, Rivory J (2003) Spectroscopic ellipsometry study of a self-organized Ge dot layer. J Appl Phys 94:2248-2253. doi: $10.1063 / 1.1592882$

Garcia-Caurel E, De Martino A, Drévillon B (2004) Spectroscopic Mueller polarimeter based on liquid crystal devices. Thin Solid Films 455:120-123. doi:10.1016/j.tsf. 2003.12.056

Giangregorio MM, Losurdo M, Sacchetti A, Capezzuto P, Bruno G (2006) Plasma processing of the $\mathrm{Si}(001)$ surface for tuning SPR of $\mathrm{Au} / \mathrm{Si}$-based plasmonic nanostructures. J Lumin 121:322-326. doi:10.1016/j.jlumin.2006.08.084

Goletti C, Bussetti G, Arciprete F, Chiaradia P, Chiarotti G (2002) Infrared surface absorption in $\mathrm{Si}(111) 2 \times 1$ observed with reflectance anisotropy spectroscopy. Phys Rev B 66: 153307. doi:10.1103/PhysRevB.66.153307

Gouadec G, Colomban P (2007) Raman Spectroscopy of nanomaterials: how spectra relate to disorder, particle size and mechanical properties. Prog Cryst Growth Charact Mater 53:1-56. doi:10.1016/j.pcrysgrow.2007.01.001

Grunthaner FJ, Grunthaner PJ (1986) Chemical and electronic structure of the $\mathrm{SiO}_{2} / \mathrm{Si}$ interface. Mater Sci Rep 1:65-160; and references therein. doi:10.1016/S0920-2307(86)80001-9

Haes AJ, Zou S, Schatz GC, van Duyne RP (2004) A nanoscale optical biosensor: the long range distance dependence of the localized surface plasmon resonance of noble metal nanoparticles. J Phys Chem B 108:109-116. doi:10.1021/ jp0361327

Hall AC (1966) On the use of ellipsometry for adsorption measurements below monolayer coverage. J Phys Chem 70:1702-1704. doi:10.1021/j100878a003 
Harrison MT, Kershaw SV, Burt MG, Rogach A, Eychmüller A, Weller H (1999) Investigation and factors affecting the photoluminescence of colloidally-prepared $\mathrm{HgTe}$ nanocrystals. J Mater Chem 9:2721-2723. doi:10.1039/a907 $224 \mathrm{a}$

Hens Z, Vanmaekelbergh D, Kooij ES, Wormeester H, Allan G, Delerue C (2004) Effect of quantum confinement on the dielectric function of PbSe. Phys Rev Lett 92:026808. doi:10.1103/PhysRevLett.92.026808

Hilfiker JN, Singh N, Tiwald T, Convey D, Smith SM, Baker JH, Tompkins HG (2008) Survey of methods to characterize thin absorbing films with spectroscopic ellipsometry. Thin Solid Films 516:7979-7989. doi:10.1016/j.tsf. 2008.04.060

Himpsel FJ, Altmann KN, Bennewitz R, Crain JN, Kirakosian A, Lin JL, McChesney JL (2001) One-dimensional electronic states at surfaces. J Phys Condens Matter 13: 11097-11115; and references therein. doi:10.1088/0953$8984 / 13 / 49 / 301$

Horiba Jobin Yvon (2005) How the MM-16 spectroscopic ellipsometer works. Technical note. http://www.horiba.com/ scientific/products/ellipsometers/thin-film-metrology/spectro scopic-ellipsometers/?L $=0$

Horiba Jobin Yvon (2008) AutoSE, the simplest thin film measurement tool ever! Technical note. http://www. horiba.com/scientific/products/ellipsometers/thin-filmmetrology/spectroscopic-ellipsometers/?L=0

Huang H-T, Terry FL Jr (2004) Spectroscopic ellipsometry and reflectometry from gratings (scatterometry) for critical dimension measurement and in situ, real-time process monitoring. Thin Solid Films 455-456:828-836. doi:10.1016/ j.tsf.2004.04.010

Humlíček J (2008) In-situ spectroscopic ellipsometry: optimization of monitoring and closed-loop-control procedures. Phys Status Solidi A 205:793-796. doi:10.1002/pssa.2007 77798

ITRS (2007) http://www.itrs.net/Links/2007ITRS/2007_Chapters/ 2007_Metrology.pdf

Jellison GE, Modine FA (1997) Two-modulator generalized ellipsometry: theory. Appl Opt 36:8190-8198. doi:10.1364/ AO.36.008190

Jellison GE, Chisholm MF, Gorbatkin M (1993) Optical functions of chemical vapor deposited thin-film silicon determined by spectroscopic ellipsometry. Appl Phys Lett 62:3348-3350. doi:10.1063/1.109067

Kanagawa T, Hobara R, Matsuda I, Tanikawa T, Natori A, Hasegawa S (2003) Anisotropy in conductance of a quasione-dimensional metallic surface state measured by a square micro-four-point probe method. Phys Rev Lett 91:036805. doi:10.1103/PhysRevLett.91.036805

Kim TW, Choo DC, Lee DU, Lee HS, Jang MS, Park HL (2002) Coalescence and electron activation energy in CdTe/ZnTe nanostructures. Appl Phys Lett 81:487-489. doi:10.1063/1.1490634

Kooij ES, Wormeester H, Martijn Brouwer EA, van Vroonhoven E, van Silfhout A, Poelsema B (2002) Optical characterization of thin colloidal gold films by spectroscopic ellipsometry. Langmuir 18:4401-4413. doi: 10.1021/la0256127
Kreibig U (1978) Transition cluster-solid state in small gold particles. Solid State Commun 28:767-769. doi:10.1016/ 0038-1098(78)91341-8

Kreibig U, Vollmer M (1995) Optical properties of metal clusters. Springer, Berlin

Laskarakis A, Logothetidis S, Pavlopoulou E, Gioti M (2004) Mueller matrix spectroscopic ellipsometry: formulation and application. Thin Solid Films 455-456:43-49. doi:10.1016/ j.tsf.2003.11.197

Lautenschlager P, Garriga M, Vina L, Cardona M (1987) Temperature dependence of the dielectric function and interband critical points in silicon. Phys Rev B 36:48214830. doi:10.1103/PhysRevB.36.4821

Lee DS, Kim WS, Choi SH, Kim J, Lee HW, Lee JH (2005) Characterization of $\mathrm{ZrO}_{2}$ co-doped with $\mathrm{Sc}_{2} \mathrm{O}_{3}$ and $\mathrm{CeO}_{2}$ electrolyte for the application of intermediate temperature SOFCs. Solid State Ionics 176:33-39. doi:10.1016/j.ssi. 2004.07.013

López-Lozano X, Stekolnikov A, Furthmüller J, Bechstedt F (2005) Band structure and electron gas of In chains on Si(111). Surf Sci 589:77-90. doi:10.1016/j.susc.2005.05. 053

Losurdo M (2004) Relationships among surface processing at the nanometer scale, nanostructure and optical properties of thin oxide films. Thin Solid Films 455-456:301-312. doi:10.1016/j.tsf.2003.11.196

Losurdo M, Giangregorio MM, Capezzuto P, Bruno G, Cerqueira MF, Stepikova M (2003) Dielectric function of nanocrystalline silicon with few nanometers $(<3 \mathrm{~nm})$ grain size. Appl Phys Lett 82:2993-2995. doi:10.1063/1.1569 052

Losurdo M, Bruno G, Kim TH, Choi S, Brown A, Moto A (2005) Nucleation and growth mode of the molecular beam epitaxy of $\mathrm{GaN}$ on $4 \mathrm{H}-\mathrm{SiC}$ exploiting real time spectroscopic ellipsometry. J Cryst Growth 284:156-165. doi:10.1016/ j.jcrysgro.2005.07.016

Maier SA (2007) Plasmonics: fundamentals and applications. Springer, New York

Marabelli F, Wachter P (1987) Covalent insulator $\mathrm{CeO}_{2}$ : optical reflectivity measurements. Phys Rev B 36:1238-1243. doi: 10.1103/PhysRevB.36.1238

Maxwell-Garnett JC (1904) Colours in metal glasses and in metallic films. Philos Trans R Soc Lond 203:385-420. doi:10.1098/rsta.1904.0024

McIntyre JD, Aspnes DE (1971) Differential reflection spectroscopy of very thin surface films. Surf Sci 24:417-434. doi:10.1016/0039-6028(71)90272-X

Moharam MG, Gaylord TK (1981) Rigorous coupled analysis of planar-grating diffraction. J Opt Soc Am 71:811-818. doi:10.1364/JOSA.71.000811

Moharam MG, Grann EB, Pommet DA, Gaylord TK (1995) Formulation for stable and efficient implementation of the rigorous coupled-wave analysis of binary gratings. J Opt Soc Am A 12:1068-1076. doi:10.1364/JOSAA.12.001068

Nerbø IS, Kildemo M, Le Roy S, Simonsen I, Søndergård E, Holt L, Walmsley J (2008) Characterization of nanostructured GaSb: comparison between large-area optical and local direct microscopic techniques. Appl Opt 47: 5130-5139. doi:10.1364/AO.47.005130

Nimtz G (1982) In: Hellwege K-H (ed) Landolt-Börnstein, vol 17b. Springer-Verlag, Berlin, p 239 
Novikova T, De Martino A, Ben Hatit S, Drévillon B (2006) Application of Mueller polarimetry in conical diffraction for $\mathrm{CD}$ measurements in microelectronics. Appl Opt 45: 3688-3697. doi:10.1364/AO.45.003688

Novikova T, De Martino A, Bulkin P, Nguyen Q, Drévillon B, Popov V, Chumakov A (2007) Metrology of replicated diffractive optics with Mueller polarimetry in conical diffraction. Opt Express 15:2033-2046. doi:10.1364/OE.15. 002033

Oates TWH (2006) Real time spectroscopic ellipsometry of nanoparticle growth. Appl Phys Lett 88:213115. doi: 10.1063/1.2206870

Ordal MA, Long LL, Bell RJ, Bell SE, Bell RR, Alexander JRW, Ward CA (1983) Optical properties of the metals $\mathrm{Al}, \mathrm{Co}, \mathrm{Cu}, \mathrm{Au}, \mathrm{Fe}, \mathrm{Pb}, \mathrm{Ni}, \mathrm{Pd}, \mathrm{Pt}, \mathrm{Ag}, \mathrm{Ti}$, and $\mathrm{W}$ in the infrared and far infrared. Appl Opt 22:1099-1119. doi: 10.1364/AO.22.001099

Patil S, Seal S, Guo Y, Schulte A, Norwood J (2006) Role of trivalent $\mathrm{La}$ and $\mathrm{Nd}$ dopants in lattice distortion and oxygen vacancy generation in cerium oxide nanoparticles. Appl Phys Lett 88:243110-243113. doi:10.1063/1.2210 795

Patsalas P, Logothetidis S, Sygellou L, Kennou S (2003) Structure-dependent electronic properties of nanocrystalline cerium oxide films. Phys Rev B 68:035104-035113. doi:10.1103/PhysRevB.68.035104

Press WH, Flannery BP, Teukolsky SA, Vetterling WT (1992) Numerical recipes in $\mathrm{C}$ : second edition, the art of scientific computing. Cambridge University Press, Cambridge

Price J, Lysaght PS, Song SC, Li H-J, Diebold AC (2007) Identification of sub-band-gap absorption features at the $\mathrm{HfO}_{2} / \mathrm{Si} 100$ interface via spectroscopic ellipsometry. Appl Phys Lett 91:061925. doi:10.1063/1.2769389

Quintanilha R (2005) Etude du problème inverse en diffractométrie spectroscopique. $\mathrm{PhD}$ Thesis, Institut National Polytechnique de Grenoble

Rath S (2005) Depth profiling of charging effect of Si nanocrystals embedded in $\mathrm{SiO}_{2}$. J Metastable Nanocryst Mater 23:125-130. doi:10.4028/www.scientific.net/JMNM.23.125

Rogach A, Kershaw S, Burt M, Harrison M, Kornowski A, Eychmüller A, Weller H (1999) Colloidally prepared HgTe nanocrystals with strong room-temperature infrared luminescence. Adv Mater 11:552-555. doi:10.1002/(SICI) 1521-4095(199905)11:7<552::AID-ADMA552>3.0.CO; 2-Q

Roither J, Kovalenko MV, Pichler S, Schwarzl T, Heiss W (2005) Nanocrystal-based microcavity light-emitting devices operating in the telecommunication wavelength range. Appl Phys Lett 86:241104. doi:10.1063/1.1947888

Rossow U (1995) In: McGilp JF (ed) Epioptics, chap 3. Springer-Verlag, Berlin, p 46

Santos PV, Etchegoin P, Cardona M, Brar B, Kroemer H (1994) Optical anisotropy in InAs/AlSb superlattices. Phys Rev B 50:8746-8754. doi:10.1103/PhysRevB.50.8746

Scaffardi LB, Tocho JO (2006) Size dependence of refractive index of gold nanoparticles. Nanotechnology 17:13091315. doi:10.1088/0957-4484/17/5/024

Sendelbach M, Archie CN (2003) Scatterometry measurement precision and accuracy below $70 \mathrm{~nm}$. In Metrology, inspection, and process control for microlithography XVII. Proceedings SPIE, vol 5038, pp 224-238. doi:10.1117/ 12.488117

Shkrebtii AI, Esser N, Richter W, Schmidt WG, Bechstedt F, Kley A, Fimland BO, Del Sole R (1998) Reflectance anisotropy of GaAs(100). Theory experiment. Phys Rev Lett 81:721-724. doi:10.1103/PhysRevLett.81.721

Shopova SI, Farca G, Rosenberger AT, Wickramanayake WMS, Kotov NA (2004) Microsphere whispering-gallerymode laser using HgTe quantum dots. Appl Phys Lett 85:6101-6103. doi:10.1063/1.1841459

Sin A, Dubitsky Y, Zaopo A, Aricò AS, Gullo L, La Rosa D, Siracusano S, Antonucci V, Oliva C, Ballabio O (2004) Preparation and sintering of $\mathrm{Ce}_{1-x} \mathrm{Gd}_{x} \mathrm{O}_{2-x / 2}$ nanopowders and their electrochemical and EPR characterization. Solid State Ionics 175:361-366. doi:10.1016/j.ssi.2004.03.034

Teboul E (2008) Innovations in ellipsometry facilitates thin film analysis. Laser Focus World, pp 76-79

Tompkins HG (1993) A user's guide to ellipsometry. Academic Press, New York

Tompkins HG, Irene EA (2005) In: Handbook of ellipsometry. William Andrew/Springer, New York

Toudert J, Babonneau D, Simonot L, Camelio S, Girardeau T (2008) Quantitative modelling of the surface plasmon resonances of metal nanoclusters sandwiched between dielectric layers: the influence of nanocluster size, shape and organization. Nanotechnology 19:125709-125710 and reference therein

Tsunekawa S, Sahara R, Kawazoe Y, Kasuya A (2000) Origin of the blue shift in ultraviolet absorption spectra of nanocrystalline $\mathrm{CeO}(2-\mathrm{x})$ particles. Mater Trans 41:1104-1107

Ukraintsev V (2006) A comprehensive test of optical scatterometry readiness for $65 \mathrm{~nm}$ technology production. In: Metrology, inspection, and process control for microlithography XX. Proceedings SPIE, vol 6152, p $61521 \mathrm{G}$. doi: $10.1117 / 12.657649$

Wang Y, Irene EA (2000) Consistent refractive index parameters for ultrathin $\mathrm{SiO}_{2}$ films. J Vac Sci Technol B 18: 279-282. doi:10.1116/1.591183

Wasserman SR, Whitesides GM, Tidswell IM, Ocko BM, Pershan PS, Axe JD (1989) The structure of self assembled monolayers of alkylsiloxanes on silicon: a comparison of results from ellipsometry and low angle X-ray reflectivity. J Am Chem Soc 111:5852-5861. doi:10.1021/ ja00197a054

Wessels JM, Nothover HG, Ford WE, von Wrochem F, Scholz F, Vossmeyer T, Schroedter A, Weller H, Yasuda A (2004) Optical and electrical properties of three-dimensional interlinked gold nanoparticle assemblies. J Am Chem Soc 126:3349-3356. doi:10.1021/ja0377605

Wormeester H, Henry AI, Kooij ES, Poelsema B, Pileni MP (2006) Ellipsometric identification of collective optical properties of silver nanocrystal arrays. J Chem Phys 124: 204713-204719. doi:10.1063/1.2200647

Wu PC, Losurdo M, Kim TH, Choi S, Bruno G, Brown AS (2007) In situ spectroscopic ellipsometry to monitor surface plasmon resonant group-III metals deposited by molecular beam epitaxy. J Vac Sci Technol B 25:1019-1023. doi: $10.1116 / 1.2734163$ 
Wu PC, Losurdo M, Kim TH, Giangregorio M, Bruno G, Everitt HO, Brown AS (2009) Plasmonic gallium nanoparticles on polar semiconductors: interplay between nanoparticle wetting, localized surface plasmon dynamics, and interface charge. Langmuir 25(2):924-930. doi:10.1021/la802678y

Yeom HW, Takeda S, Rotenberg E, Matsuda I, Horikoshi K, Schaefer J, Lee CM, Kevan SD, Ohta T, Nagao T, Hasegawa S (1999) Instability and charge density wave of metallic quantum chains on a silicon surface. Phys Rev Lett 82:4898-4901. doi:10.1103/PhysRevLett.82.4898

Yu PY, Cardona M (1999) Fundamentals of semiconductors, 2nd edn. Springer-Verlag, Berlin

Zhao X, Wei CM, Yang L, Chou MY (2004) Quantum confinement and electronic properties of silicon nanowires. Phys Rev Lett 92:236805. doi:10.1103/PhysRevLett.92.236805 